\title{
Hepatocellular carcinoma-targeted nanoparticles for cancer therapy
}

\author{
CHIEN-HSUN WU ${ }^{1}$, CHUN-HSIN LAN $^{1}$, KUAN-LIN WU ${ }^{1}$, YAO-MING WU ${ }^{2}$, \\ WANN-NENG JANE ${ }^{3}$, MICHAEL HSIAO ${ }^{4}$ and HAN-CHUNG WU ${ }^{1,4}$ \\ ${ }^{1}$ Institute of Cellular and Organismic Biology, Academia Sinica, Taipei 115; \\ ${ }^{2}$ Department of Surgery, National Taiwan University Hospital, Taipei 100; \\ ${ }^{3}$ Institute of Plant and Microbial Biology, ${ }^{4}$ Genomics Research Center, \\ Academia Sinica, Taipei 115, Taiwan, R.O.C.
}

Received August 7, 2017; Accepted October 20, 2017

DOI: $10.3892 /$ ijo.2017.4205

\begin{abstract}
Nanocarriers, such as liposomes, have the potential to increase the payload of chemotherapeutic drugs while decreasing toxicity to non-target tissues; such advantageous properties can be further enhanced through surface conjugation of nanocarriers with targeting moieties. We previously reported that SP94 peptides, identified by phage display, exhibited higher binding affinity to human hepatocellular carcinoma (HCC) than to hepatocytes and other normal cells. Here, we confirm the tumor-targeting properties of SP94 peptide by near-infrared fluorescence imaging. Non-targeted PEGylated liposomal doxorubicin (LD) and SP94-conjugated PEGylated liposomal doxorubicin (SP94-LD) were compared by assessing pharmacokinetics, tissue distribution, and antitumor efficacy in xenograftbearing mice, in order to investigate the effectiveness of SP94-mediated targeting for cancer therapy. SP94-LD demonstrated a significant increase in drug accumulation in tumors, while its plasma residence time was the same as its non-targeted equivalent. Consistent with this result, conjugation of targeting peptide SP94 enhances the therapeutic efficacy of liposomal doxorubicin in mouse models with hepatocellular carcinoma xenografts. Furthermore, combination targeted therapy exhibited a significant enhancement against orthotopic tumor growth, and markedly extended the survival of mice compared with all other treatments. Our study shows that SP94-mediated targeting enhances antitumor efficacy by improving tumor pharmacokinetics and tissue distribution, allowing large amounts of antitumor drugs to accumulate in tumors.
\end{abstract}

Correspondence to: Dr Han-Chung Wu, Institute of Cellular and Organismic Biology, Academia Sinica, 128 Academia Road, Section 2, Nankang, Taipei 11529, Taiwan, R.O.C.

E-mail: hcw0928@gate.sinica.edu.tw

Key words: hepatocellular carcinoma, drug delivery system, pharmacokinetics, biodistribution, ligand-targeted therapeutics

\section{Introduction}

Lipid nanoparticles have been widely used as pharmaceutical carriers to increase the efficacy of chemotherapeutics (1). These drug carriers are expected to passively accumulate in tumors with leaky vasculature, via the enhanced permeability and retention (EPR) effect (2). One successful example is the reformulation of doxorubicin encapsulated into PEGylated liposomes. Doxorubicin is an anthracycline widely used in the treatment of various cancers, including metastatic breast cancer, ovarian cancer, and AIDS-related Kaposi's sarcoma; however, its efficacy is limited by its toxicity. Liposomal formulation enhances the therapeutic index of anticancer drugs, either by improving the pharmacokinetic and pharmacodynamic profiles, or by decreasing the exposure of normal host tissues.

In order to improve the specificity of nanocarriers, targeting ligands may be attached to their surface via a PEG spacer arm. The targeting ligands, which are attached to the distal end of the spacer arm on the surface of nanocarriers, facilitate access of the carrier to the targeted site of interaction (3). Delivery of nanoparticle involving the use of peripherally-conjugated targeting moieties is known as active targeting. Active targeting is a promising tool for the treatment of cancer due to its ability to increase therapeutic effectiveness and reduce potential side effects. Active tumor targeting has been achieved using various targeting ligands, including antibodies (4) and their molecular fragments (5), nucleic acids aptamers (6), and small molecules: vitamins $(7,8)$, peptides $(9-12)$, and carbohydrates (13). The targeted form of PEGylated liposomal doxorubicin is expected to become a major part of the next generation of this therapeutic modality.

The SP94 peptide (SFSIIHTPILPL), a targeting ligand isolated from phage-displayed selections $(10,14)$, was reported to possess high and specific affinity for various human hepatocellular carcinoma cell lines, and exhibit minimal interaction with healthy hepatocytes and other tissues $(10,14)$. At the present time, the antigen recognized by SP94 has not been identified, but it has been immunohistochemically characterized. SP94 can bind to tumor cells in surgical specimens of hepatocellular carcinoma, but not to their normal 
counterparts. The unknown target molecule recognized by SP94 was reported to be expressed in $\sim 60 \%$ of patients with hepatocellular carcinoma (10).

In recent years, additional tumor-targeted delivery platforms modified with SP94 peptide are also being developed, including mesoporous silica nanoparticle-supported lipid bilayers (protocells) $(14,15)$, bacteriophage MS2 virus-like particles (VLPs) (16) and HCC targeting probe $\left({ }^{99 \mathrm{~m}} \mathrm{Tc} /{ }^{188} \mathrm{Re}-\right.$ HYNIC-SP94) for imaging and therapy (17). Multivalent binding of SP94 peptide results in a 10,000-fold greater avidity for human hepatocellular carcinoma than for hepatocytes, endothelial cells, peripheral blood mononuclear cells, B-lymphocytes, or T-lymphocytes (14). Exquisite targeting specificity of SP94 peptide combined with enhanced tumor delivery of multicomponent cargos permits sensitive discrimination between target and normal tissue. Thus, SP94 peptide is an ideal model with which to investigate the mechanism of active tumor targeting.

The potential of SP94 in drug delivery was subsequently evaluated using SP94-conjugated, doxorubicin-encapsulated liposomes. It was previously shown that SP94-LD is more effective than non-targeted LD in treating mice with xenografts of human hepatocellular carcinoma (10). SP94-targeted PEGylated liposomal doxorubicin (SP94-LD) is believed to accumulate around cancerous tissue (via the EPR effect) and bind to the cancer cell surface, then being internalized by ligand-mediated endocytosis (via active targeting effect) (18). However, the role of active targeting in nanoparticle delivery is controversial, and it is difficult to predict how a targeted nanoparticle drug will behave in vivo. Herein, we confirm the mechanisms underlying the enhanced cellular uptake of SP94-modified nanoparticles, and moreover, investigate the contribution of SP94 peptide during the cellular internalization of SP94-targeted nanoparticles.

To date, the only chemotherapeutic agent to exert a survival benefit in patients with hepatocellular carcinoma is sorafenib $(19,20)$. In order to evaluate the feasibility of introducing SP94-targeted nanomedicine into clinical trials against liver cancer, we examined the pharmacokinetic profile, biodistribution, and in vivo antitumor activity of SP94-targted nanomedicine against hepatocellular carcinoma, and compared these properties to those of free drugs and non-targeted liposomal drugs. Furthermore, we found synergistic/additive growth inhibition by combination of doxorubicin and vinorelbine in HCC cell lines in vitro and in vivo. For in vivo evaluation, we developed an orthotopic hepatocellular carcinoma model to recapitulate the tumor growth pattern observed in liver cancer patients, and used this model to study the influence of the liver microenvironment on response to the combination therapy.

\section{Materials and methods}

Cell lines and cell culture. The Mahlavu and SK-HEP-1 human hepatocellular carcinoma lines were used in this study. The cell lines were maintained in DMEM and $10 \%$ fetal bovine serum at $37^{\circ} \mathrm{C}$ in a humidified atmosphere of $5 \% \mathrm{CO}_{2}$ in air.

Peptide synthesis and labeling. Targeting SP94(SFSIIHTPILPL) peptides were synthesized and purified by reverse-phase high-performance liquid chromatography to $>95 \%$ purity by the Peptide Synthesis Core Facility, Institute of Cellular and Organismic Biology, Academia Sinica. The predicted mass was confirmed by mass spectrometry.

Synthesis of peptide-PEG-DSPE conjugates. A total of $8.5 \mathrm{mg}$ of NHS-PEG-DSPE [N-hydroxysuccinimido-carboxylpolyethyleneglycol(MW,3400)-derived distearoylphosphatidyl ethanolamine] (NOF Corp.) dissolved in $0.25 \mathrm{ml}$ of dichloromethane (Sigma-Aldrich) was added to $0.25 \mathrm{ml}$ of DMSO (Sigma-Aldrich) containing $3.1 \mathrm{mg}$ of peptide. This was then mixed with $0.011 \mathrm{ml}$ of triethylamine (Sigma-Aldrich) to catalyze the reaction. The stoichiometric molar ratio of peptide and NHS-PEG-DSPE was 1.1:1. The reaction was carried out for $72 \mathrm{~h}$ at room temperature. The peptide-PEGDSPE conjugates were purified by dialysis with a $2-\mathrm{kDa}$ cut-off membrane (Spectrum), and were then dried through lyophilization.

Preparation of peptide-liposomal drugs. A lipid film hydration method was used to prepare PEGylated liposomes composed of distearoylphosphatidylcholine, cholesterol, and mPEG2000-DSPE, which were then used to encapsulate doxorubicin (3:2:0.3 molar ratio) or vinorelbine (3:2:0.15 molar ratio). The lipid films were hydrated at $60^{\circ} \mathrm{C}$ in $250 \mathrm{mM}$ ammonium sulfate or $300 \mathrm{mM}$ ammonium salts of 5-sulfosalicylic acid solution, and were extruded through polycarbonate membrane filters with a pore size of $0.1 \mu \mathrm{m}$ using high-pressure extrusion equipment (Lipex Biomembranes, Vancouver, BC, Canada) at $55^{\circ} \mathrm{C}$. Doxorubicin or vinorelbine were encapsulated by a remote loading method, at concentrations of $1 \mathrm{mg}$ or $3.5 \mathrm{mg}$ per $10 \mu \mathrm{mol}$ of phospholipid, respectively. The final concentration of liposome was estimated by phosphate assay. The peptide-PEG-DSPE was subsequently incorporated into pre-formed liposomes by co-incubation at $60^{\circ} \mathrm{C}$, the transition temperature of the lipid bilayer, for $0.5 \mathrm{~h}$ with gentle shaking. Sepharose 4B (GE Healthcare) gel filtration chromatography was used to remove released free drug, unconjugated peptides, and unincorporated conjugates. Doxorubicin concentrations in the fractions of eluent were determined by measuring fluorescence at $\lambda \mathrm{Ex} / \mathrm{Em}=485 / 590 \mathrm{~nm}$ using a spectrofluorometer (Spectra Max M5, Molecular Devices). Vinorelbine concentrations were determined using the HPLC method.

Bacteriophage preparation and labeling. M13 bacteriophages were amplified in Escherichia coli, and phage titers were determined according to published procedures. Following titer determination, the bacteriophages were simultaneously labeled with succinimidyl esters of HiLyte Fluor ${ }^{\mathrm{TM}} 750$ using a modified procedure $(21,22)$. Briefly, HiLyte Fluor 750 succinimidyl ester was added to M13 bacteriophage in $100 \mu \mathrm{M}$ bicarbonate buffer, $\mathrm{pH}$ 8.3. The resulting solution was incubated for $1 \mathrm{~h}$ at room temperature in the dark. Following incubation, the labeled bacteriophage was precipitated by addition of a PEG-8000/2.5 M NaCl solution, and then left to stand on ice for $30 \mathrm{~min}$. The bacteriophage was pelleted by centrifugation at $10,000 \mathrm{x} \mathrm{g}$ for $15 \mathrm{~min}$. After removal of the supernatant, the pellet was resuspended in DPBS buffer. Typical dye labeling using this procedure resulted in 400-500 copies of each dye per bacteriophage particle. 
In vivo near-infrared fluorescence imaging. A total of $4 \times 10^{11}$ pfu of HiLyte Fluor 750-labeled PC94 phage was diluted in $100 \mu \mathrm{l}$ saline solution. The saline solution was injected i.v. into mice bearing subcutaneous Mahlavu tumors. A control solution of $4 \times 10^{11}$ pfu of the HiLyte Fluor 750-labeled control phage was injected into NOD.CB17-Prkd $c^{\text {scid }} / \mathbf{J}$ mice bearing subcutaneous Mahlavu tumors. In vivo imaging was performed using a Xenogen IVIS $^{\circledR}$ Imaging System 200. The animal was imaged at $0.1,0.5,6,24$, and $48 \mathrm{~h}$ post-injection using a Indocyanine Green (ICG) Filter set (excitation 710-760 nm, emission 810-875 $\mathrm{nm}$ ). Organs were dissected and imaged $48 \mathrm{~h}$ after injection of conjugate.

Animal model for in vivo targeting assay. The dorsolateral flanks of severe combined immunodeficient mice, NOD. CB17-Prkdc scid/J (4-6 weeks old), were injected s.c. with $5 \times 10^{6}$ Mahlavu or SK-HEP-1 cells. Tumors were measured with calipers, and mice were weighed twice weekly. The tumor volumes were calculated using the following formula: length $\mathrm{x}$ (width $)^{2} \times 0.52$. All animals were cared for in a specific pathogen-free room and treated in accordance with the animal care protocol approved by the Academia Sinica Animal Committee (approval no. 11-06-190).

Quantitative analysis. Plasma, brain, heart, lung, liver, kidney, or tumor samples were processed using the extraction procedure, and then analyzed using the method described by Laginha et al (23). Result concentrations were determined relative to the respective calibration curves.

Terminal deoxynucleotidyl transferase-mediated dUTP nick end labeling (TUNEL). Frozen tumor tissue sections were incubated with TUNEL reaction mixture (Roche Diagnostics) at $37^{\circ} \mathrm{C}$ for $1 \mathrm{~h}$. The slides were counterstained with Hoechst 33258 (Molecular Probes) and mounted with mounting medium (Vector Laboratories). The slides were then visualized under a fluorescent microscope. The sections were analyzed using automated cell acquisition (TissueGnostics), and TUNEL-positive areas were quantified using MetaMorph software (Molecular Devices).

CD31 staining. The frozen tumor tissue sections were fixed with methanol/acetone (1:1), washed with PBS, and immersed in blocking buffer ( $1 \%$ bovine serum albumin in PBS), followed by incubation with rat anti-mouse CD31 (BD Pharmingen). The sections were washed with PBST0.1 (0.1\% Tween-20 in PBS), and then incubated with rabbit anti-rat antibody (Stressgen) and immersed in rhodamine-labeled goat anti-rabbit antibody solution (Jackson ImmunoResearch). The slides were counterstained with Hoechst 33258, mounted with mounting medium, and visualized under a fluorescent microscope.

Complete blood count. Blood was collected by venipuncture of the right submandibular vein of unanesthetized mice with a lancet. Samples were drawn into plastic K2 EDTA blooddrawing tubes. Complete blood counts were performed using the Abbott CELL-DYN 3700 Hematology Analyzer (Abbott Diagnostics, Abbott Park, IL, USA). The parameters assessed were as follows: white blood cell (WBC) count, neutrophil absolute count (NEU), lymphocyte absolute count (LYM), red blood cell/erythrocyte count (RBC), hemoglobin concentration (HGB), hematocrit (HCT), red cell distribution width (RDW), platelet/thrombocyte (PLT), mean platelet volume count (MPV), plateletcrit (PCT), neutrophil percentage (NEU \%), lymphocyte percentage (LYM \%), monocyte percentage (MONO \%), monocyte absolute count (MONO), mean corpuscular volume (MCV), mean corpuscular hemoglobin (MCH), mean corpuscular hemoglobin concentration (MCHC), and platelet distribution width (PDW).

Drug combination study. Mahlavu cells were seeded in 96-well plates and allowed to attach overnight. Cells were treated in 96-well format in triplicate for each drug concentration combination, and viability was assessed after 3 days of treatment using the MTT-based assay. The cytotoxicity of each drug and of their combinations was assessed by drug response matrix using the Combenefit software (24). Loewe synergy score and HSA synergy score were calculated and plotting by SynergyFinder software (25).

Orthotopic implantation of human hepatocellular carcinoma in mice. NOD.CB17-Prkdc scid/J mice were used for HCC implantation. SK-HEP-1 cells were infected with Lenti-luc virus (lentivirus containing the luciferase gene). The mice were anesthetized via i.p. injection of Avertin, 2,2,2-Tribromoethanol (Sigma Chemical Co.) at a dose of $250 \mathrm{mg} / \mathrm{kg}$. Prior to orthotopic implantation, a 1-cm laparotomy was performed, and orthotopic human hepatocellular carcinoma (HCC) was established by intrahepatic injection of $10^{5}$ SK-HEP-1-Luc cells (luciferase-expressing cells) suspended in $30 \mu \mathrm{l}$ DMEM into the left liver lobe. Post-injection bleeding and tumor cell escape were avoided by short-term local compression. The abdomen was closed using an absorbable 5-0 vicryl suture, and the skin was closed with a 5-0 proline suture. For orthotopic therapeutic studies, implanted mice were treated with different formulations of anticancer drugs. Tumor progression was monitored by bioluminescence quantification. Mouse body weight and survival rate were measured. Animal care was carried out in accordance with the guidelines of Academia Sinica, Taiwan. The experimental protocols were approved by the Academia Sinica Institutional Animal Care and Utilization Committee (approval no. 11-06-190).

Data analysis. All data were expressed as the mean \pm standard error (SEM) of at least 3 independent experiments. Statistical significance was assessed using a two-tailed Student's t-test with $\mathrm{P}<0.05$ considered to be statistically significant. Non-compartmental pharmacokinetic analysis of plasma concentrations versus time was performed with pharmacokinetic parameters using WinNonlin software version 5.2 (Pharsight, Mountain View, CA, USA).

\section{Results}

Ultrastructural analysis of in vitro trafficking of lipid nanoparticles. Intracellular trafficking is a determining factor in the therapeutic efficiency of nanoparticle-based drug delivery. In the present study, transmission electron microscopy was used to investigate the mechanism of cellular uptake of SP94-modified liposomes. TEM images of SK-HEP-1 cells 

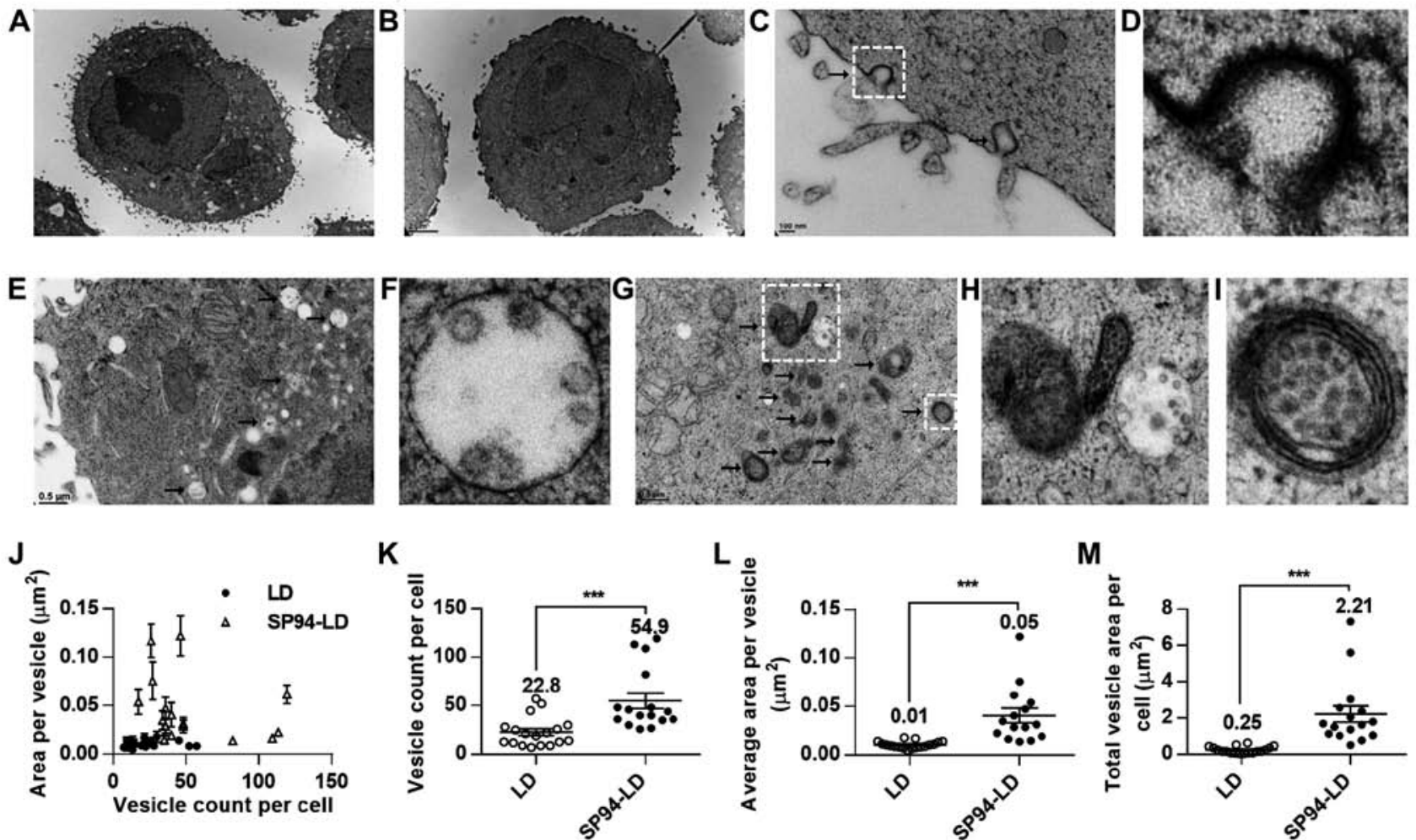

Figure 1. Transmission electron microscopic images of SP94-LD and LD internalized by SK-HEP-1 cells. The SK-HEP-1 cells (1x107 cells) were incubated with $10 \mu \mathrm{g} / \mathrm{ml} \mathrm{SP94-LD} \mathrm{or} \mathrm{LD} \mathrm{at} 37^{\circ} \mathrm{C}$ for $10 \mathrm{~min}$, and then frozen and processed for TEM. Representative electron micrographs of the cells treated with SP94-LD (A) and LD (B) are shown. (C) Coated pit structures were visually identified by the presence of the electron-dense coating of the plasma membrane (arrows). (D) High magnification image of the coated pit structures, indicated by the boxed area in (C). (E) Image of SP94-LD in endosomes (arrows). (F) High magnification image of the endosomes, as shown in (E). (G) The late endosomes deliver their cargo, SP94-LD, to the lysosomes (arrows), resulting in the electron-dense, multivesicular appearance of late endosomes known as multivesicular bodies (MVBs). (H and I) High magnification images of the regions indicated by the boxed areas in (G). TEM photomicrographs were subjected to morphometric analysis with Image $\mathbf{J}$ software. (J) Vesicle number and area distribution of cells treated with SP94-LD or LD. (K) Statistical analysis of vesicle count per cell. (L) Statistical analysis of average area per vesicle. (M) Statistical analysis of total vesicle area per cell $(n=16-18)$.

incubated with SP94-LD or LD revealed that liposomes were internalized by vesicular transport, and partially escaped to the cytosol at the perinuclear region at $37^{\circ} \mathrm{C}$ (Fig. 1A and B). After SP94-LD exposure, SK-HEP-1 cells exhibited numerous coated pit structures (Fig. 1C and D) and endocytotic vesicles in the cell membrane and cytoplasm (Fig. 1E-I). In contrast, few endocytotic vesicles were observed at the same magnification in cells incubated with LD (Fig. 1B). Analysis of the TEM images revealed that cells incubated with SP94-LD showed a high amount of internalized vesicles (Fig. 1J-M). The total vesicle area per SK-HEP-1 cell for SP94-LD was $\sim 8.8$-fold greater than that for LD (Fig. 1M).

Tumor accumulation and retention of near-infrared, fluorochrome-labeled, HCC-targeted phage. Molecular imaging plays a critical role in oncological drug development, as stated in the FDA's Critical Path Initiative documents (26). To date, no molecular imaging method has been shown to accurately detect, characterize, or monitor the response of HCC to treatment. Labeling of phages with a near-infrared fluorescence tag enables the distribution of the phages to be efficiently tracked in vivo. We evaluated the in vivo distribution of PC94 phage using a previously described optical imaging method (22). Mice bearing subcutaneous Mahlavu tumors were intravenously injected with HiLyte Fluor 750-labeled PC94 phage or control phage, and phages were monitored at 0.1 and $0.5 \mathrm{~h}$ at the initial stages, and again at 6,24 , and $48 \mathrm{~h}$ post-injection. Fig. 2A shows representative near-infrared images taken at $0.1,0.5,6,24$ and $48 \mathrm{~h}$ post-injection from two subjects. Mice injected with HiLyte Fluor 750-labeled PC94 phage exhibited higher fluorescent signals in tumors, as compared to tumors in animals injected with control phage (Fig. 2A and B).

We proceeded to further evaluate the distribution profiles of PC94 phage in mice. The accumulation of phages in Mahlavu tumor peaked at $\sim 24 \mathrm{~h}$ and then decreased gradually, but $>80 \%$ of the peak level was retained in the tumor by $48 \mathrm{~h}$ (Fig. 2B). The organ accumulation of phages in Mahlavu tumors was evaluated by near-infrared fluorescence imaging after sacrifice at $48 \mathrm{~h}$ post-injection, revealing that the SP94 peptide enhanced accumulation in tumor sites, but not healthy organs including brain, heart, lung, liver, kidney, pancreas, spleen and intestine (Fig. 2C and D).

Comparison of the pharmacokinetic and biodistribution profiles of SP94-LD and LD. For pharmacokinetic analysis, SP94-LD and LD were administered to NOD.CB17-Prkdc scid/J mice at matched $2 \mathrm{mg}$ doxorubicin/ $\mathrm{kg}$ by tail vein injection. Blood samples were withdrawn at selected time-points, and were examined for doxorubicin content using a validated fluorescent quantitative method. The blood profiles of both 
A

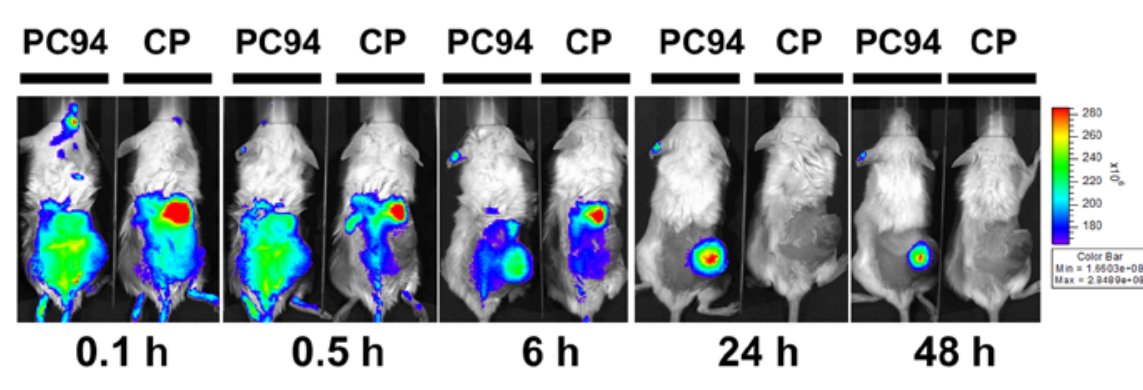

B

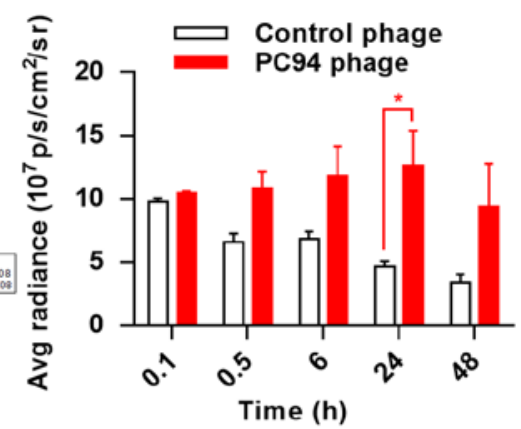

C

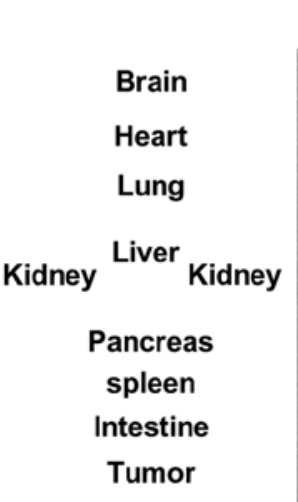

PC94

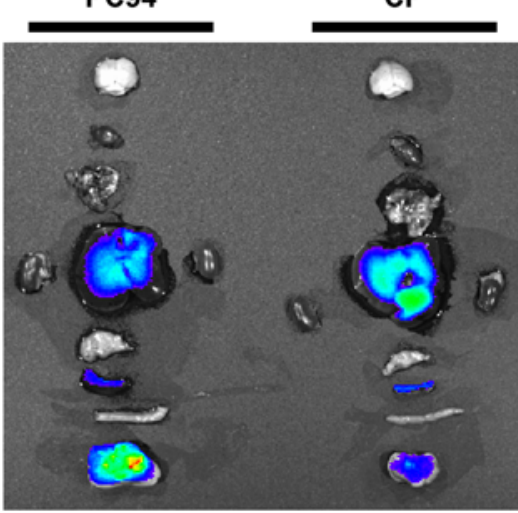

D
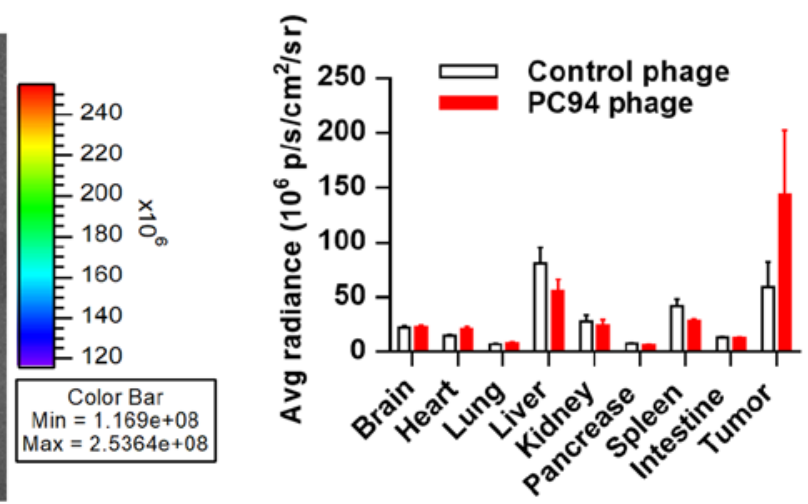

Figure 2. In vivo imaging of near-infrared, fluorochrome-labeled, HCC-targeted phage. (A) Mice bearing subcutaneous tumors (Mahlavu) were injected with HiLyte Fluor 750-labeled HCC-targeted phage (PC94) or HiLyte Fluor 750-labeled wild-type phage (Cp, control phage), and imaged at 0.1, 0.5, 6, 24, and $48 \mathrm{~h}$ after injection. (B) Quantification and kinetics of fluorochrome-labeled phage targeting. (C) Ex vivo fluorescence images of organs harvested $48 \mathrm{~h}$ after injection. (D) Fluorescence values from each organ.

SP94-LD and LD were similar throughout the study, declining progressively over time (Fig. 3A). The time course of SP94-LD and LD accumulation in tumor and various organs are shown in Fig. 3B and C. The maximum total doxorubicin concentration in tumor was $1.53 \pm 0.47 \mu \mathrm{g}$ doxorubicin/g tumor, which occurred at $24 \mathrm{~h}$ after SP94-LD administration; $1.01 \pm 0.18 \mu \mathrm{g} / \mathrm{g}$ remained at $72 \mathrm{~h}$ (Fig. 3B). Maximum tumor accumulation of LD at $2 \mathrm{mg} / \mathrm{kg}(1.03 \pm 0.69 \mu \mathrm{g} / \mathrm{g})$ occurred at $48 \mathrm{~h}$ post-injection, and gradually decreased with time to $0.89 \pm 0.69 \mu \mathrm{g} / \mathrm{g}$ at $72 \mathrm{~h}$ post-treatment. At $24 \mathrm{~h}$, tumor accumulation of SP94-LD was 1.6-fold higher than that of LD. In contrast, distribution of SP94-LD in all non-malignant tissues was similar to that of $\mathrm{LD}$ at all time-points (Fig. 3C). The tumor doxorubicin $\mathrm{AUC}_{0-72}$ for SP94-LD was $81.75 \mu \mathrm{g} \mathrm{h} / \mathrm{g}$ and the LD AUC $0-72$ was $58.23 \mu \mathrm{g} \mathrm{h} / \mathrm{g}$, representing a 1.4-fold increase in doxorubicin $\mathrm{AUC}_{0-72}$ for mice treated with the targeted drug.

Intracellular distribution and accumulation of doxorubicin. To determine the amount of bioavailable drug in tumor cells, we performed whole body perfusion through the left ventricle of the heart with DPBS before analyzing biodistribution. This operation can eliminate blood and liposomes remaining in vessels and the interstitial space of tumors. After whole body perfusion, the tumor mass, brain, heart, lung, liver, and kidneys were harvested, and doxorubicin was quantified. We used intracellular accumulation of doxorubicin as an indicator of bioavailability of the liposomal drug. SP94-LD and LD were administered to tumor (SK-HEP-1)-bearing mice at matched $1 \mathrm{mg}$ doxorubicin/kg by i.v. injection. The doxorubicin levels were measured in the blood at different time-points using a fluorescent quantitative method. The blood profiles of both SP94-LD and LD were similar (Fig. 4A). Tumor uptake of SP94-LD at $1 \mathrm{mg} / \mathrm{kg}$ gradually increased, before peaking at $0.99 \pm 0.24 \mu \mathrm{g} / \mathrm{g}$ at $24 \mathrm{~h}$ post-injection, while $0.70 \pm 0.13 \mu \mathrm{g} / \mathrm{g}$ remained at $48 \mathrm{~h}$ (Fig. 4B). Maximum tumor uptake of LD at $1 \mathrm{mg} / \mathrm{kg}(0.55 \pm 0.11 \mu \mathrm{g} / \mathrm{g})$ occurred $24 \mathrm{~h}$ post-injection, and experienced almost no change over time, remaining at $0.55 \pm 0.19 \mu \mathrm{g} / \mathrm{g}$ at $48 \mathrm{~h}$ post-injection. At $24 \mathrm{~h}$, a 1.79 -fold higher uptake of SP94-LD was observed as compared to LD. The tumor doxorubicin $\mathrm{AUC}_{0-48}$ for SP94-LD was $34.45 \mu \mathrm{g} \mathrm{h} / \mathrm{g}$ and the $\mathrm{LD} \mathrm{AUC}_{0-48}$ was $21.27 \mu \mathrm{g} \mathrm{h} / \mathrm{g}$, representing a 1.62 -fold increase in doxorubicin $\mathrm{AUC}_{0-48}$ when conjugated to SP94. The uptake of both drugs in most non-malignant tissues was low at $1 \mathrm{~h}$ post-injection; uptake of both drugs by liver and kidney gradually increased by $24 \mathrm{~h}$, and then slowly declined thereafter (Fig. 4C). Interestingly, the retention of SP94-LD in liver and kidney were lower than that of LD at $24 \mathrm{~h}$ postinjection, although these differences were not statistically significant $(\mathrm{P}=0.14$ and $\mathrm{P}=0.06$ respectively).

Efficacy of SP94-targeted liposomal doxorubicin in hepatocellular carcinoma xenograft models. To evaluate the antitumor efficacy of systemically-administered SP94-LD as compared to LD, NOD.CB17-Prkd $c^{\text {scid/J }}$ were inoculated s.c. with SK-HEP-1 tumors. Mice bearing hepatocellular carcinoma xenografts $\left(\sim 100 \mathrm{~mm}^{3}\right)$ were assigned into four groups for different treatments: A, SP94-LD; B, free doxorubicin (FD); C, LD; and $\mathrm{D}, \mathrm{PBS}$. Treatments were administered through tail vein injection, 
A

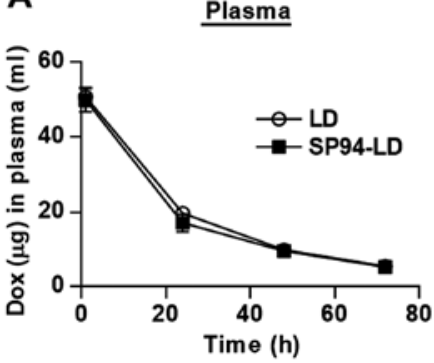

C
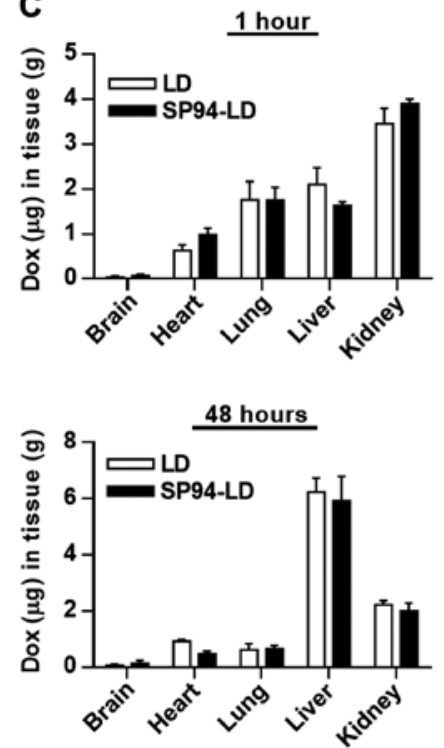

B

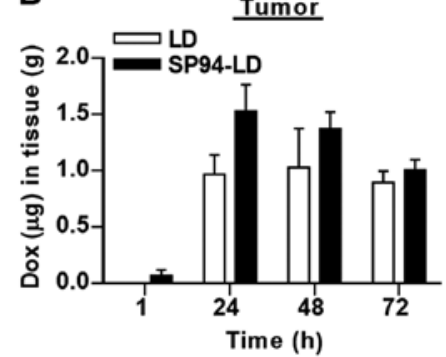

$\underline{24 \text { hours }}$

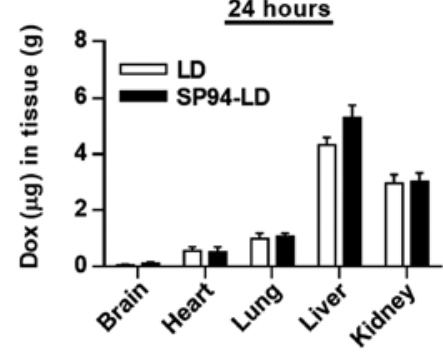

72 hours

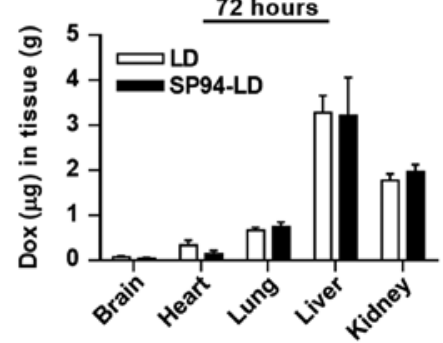

Figure 3. Pharmacokinetic profiles of liposomal doxorubicin conjugated to targeting peptide SP94 in mice bearing hepatocellular carcinoma xenografts. NOD.CB17-Prkdcsid/J mice bearing SK-HEP-1 tumors (four mice per time-point) received tail vein injections of either LD or SP94-LD at $2 \mathrm{mg} / \mathrm{kg}$. At selected time-points post injection, mice were euthanized, and whole blood and various organs were excised and analyzed for doxorubicin auto-fluorescence signals. The levels of doxorubicin were quantified with a standard curve generated from the fluorescence emission of known amounts of doxorubicin (N=4). Doxorubicin concentrations in (A) plasma and (B) tumors are shown. (C) Time course of doxorubicin distribution in the indicated organs after i.v. administration of either LD or SP94-LD at $2 \mathrm{mg} / \mathrm{kg}$. Data represent the means \pm SEM of total doxorubicin in the brain, heart, lung liver, kidney and tumor.
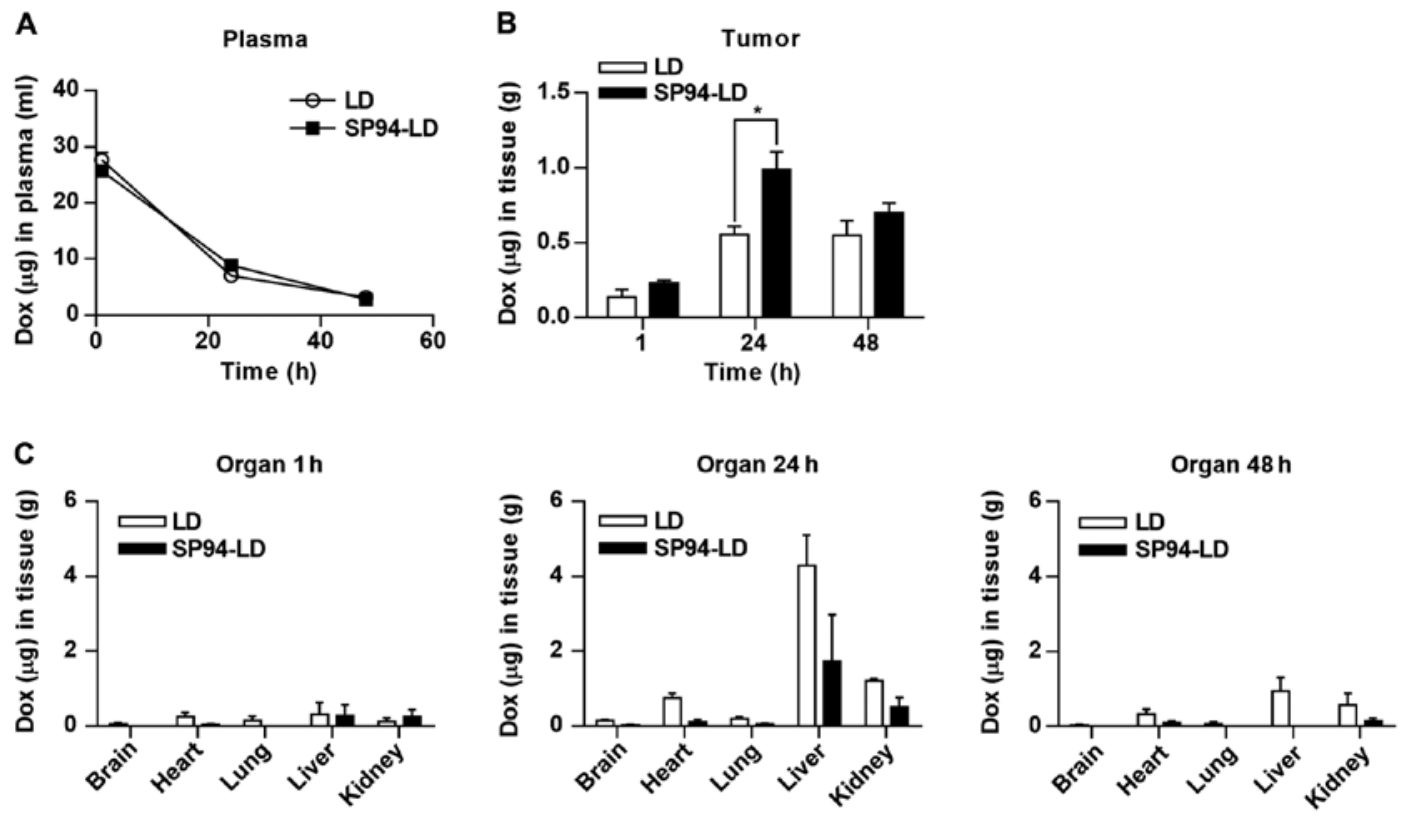

Figure 4. Conjugation of targeting peptide SP94 to liposomal doxorubicin selectively enhances drug delivery to tumor cells in vivo. NOD.CB17-Prkd ${ }^{\text {scid } / J}$ mice bearing SK-HEP-1 tumors (four mice per time-point) received tail vein injections of either LD or SP94-LD at $1 \mathrm{mg} / \mathrm{kg}$. At selected time-points post-injection, mice were euthanized. Various organs were excised after perfusion with PBS, and analyzed for auto-fluorescent doxorubicin signals. The levels of doxorubicin were quantified with a standard curve generated from the fluorescence emission of known amounts of doxorubicin ( $\mathrm{N}=4$ ). Doxorubicin concentrations in (A) plasma and (B) tumors are shown. (C) Tissue concentrations of doxorubicin in mice administered with either LD or SP94-LD at selected time-points after injection. 

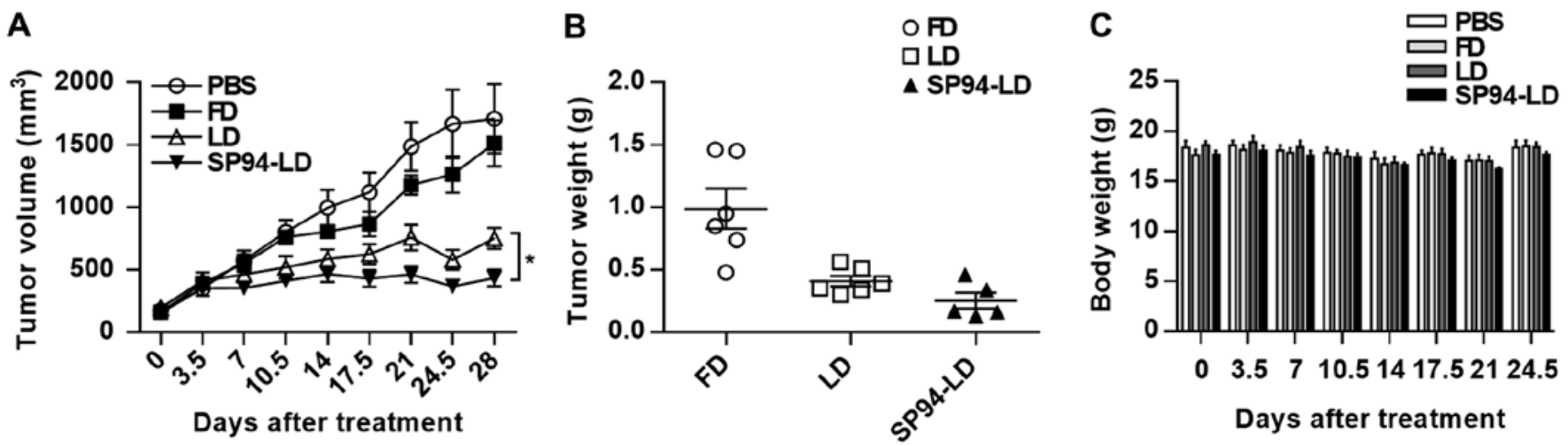

D
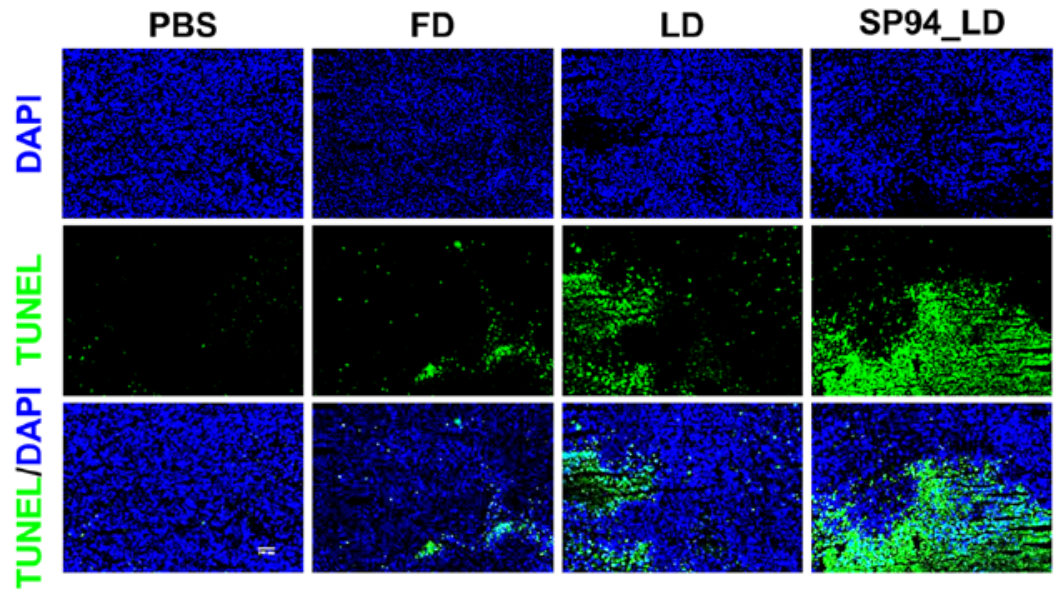

Days after treatment

$\mathbf{F}$

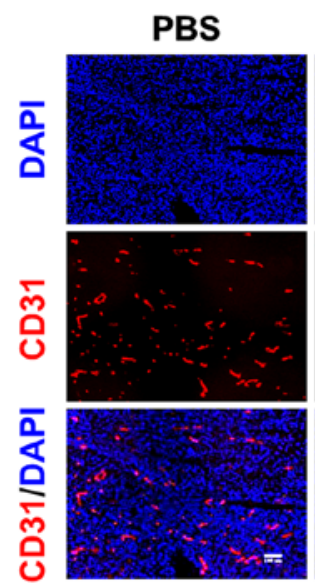

FD

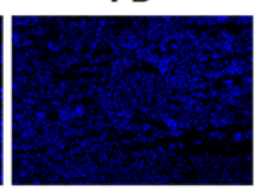

LD
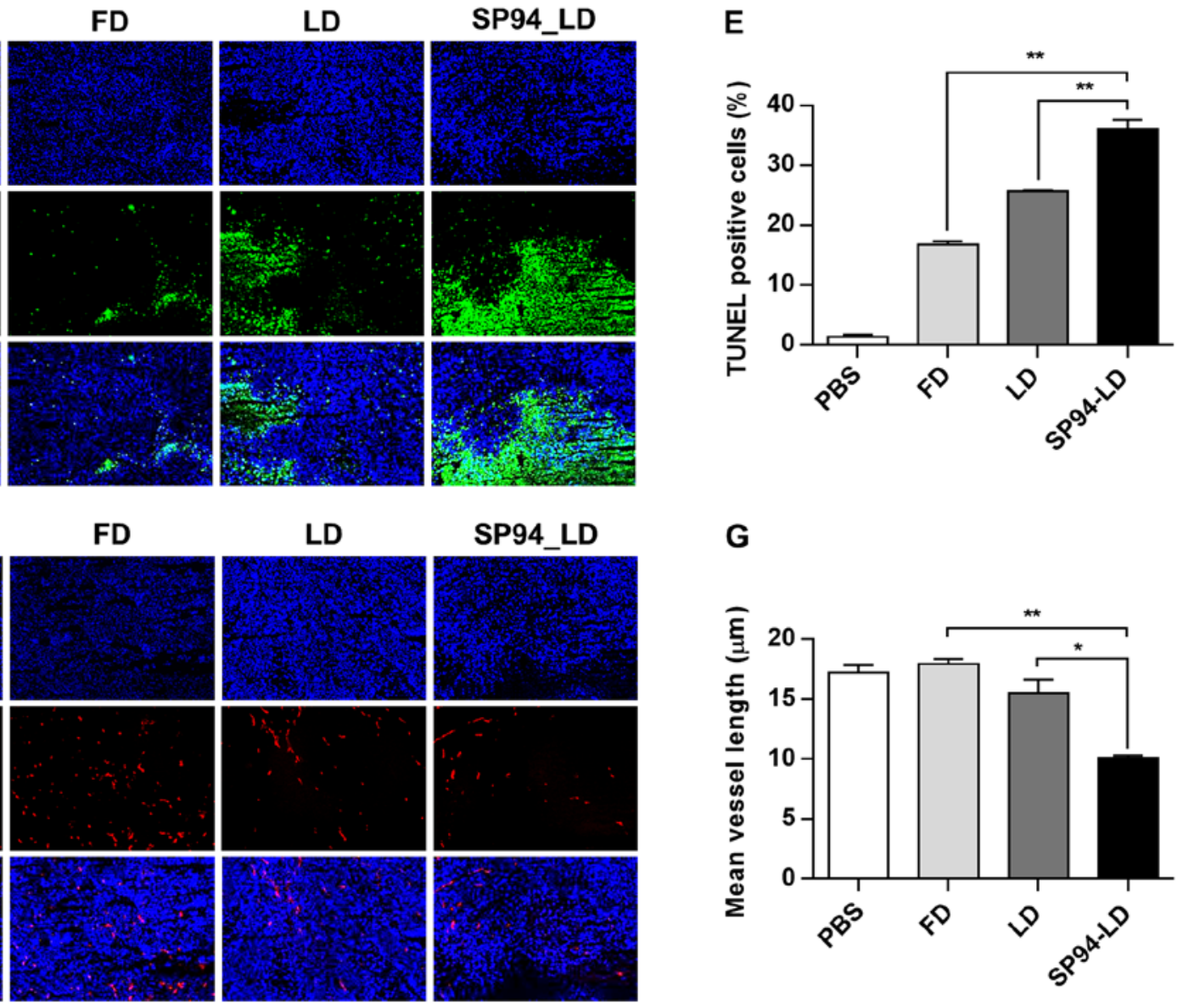

Figure 5. In vivo antitumor effects of liposomal doxorubicin conjugated to SP94 in mice bearing human hepatocellular carcinoma xenograft tumors. Mice bearing SK-HEP-1 were treated with $1 \mathrm{mg} / \mathrm{kg}$ SP94-LD, $1 \mathrm{mg} / \mathrm{kg} \mathrm{LD}, 1 \mathrm{mg} / \mathrm{kg}$ FD, or PBS. All compounds were injected twice weekly via tail vein. (A) Tumor volume of mice in each group at the indicated times ( $\mathrm{n}=6$ in each group). (B) At the end of the treatment period, tumor weight was measured. (C) Mean body weight of mice in each treatment group. (D) Frozen sections of tumor tissues from each treatment group were stained with TUNEL (green) and DAPI (blue) to visualize apoptotic tumor cells. (E) TUNEL-positive cells in each treatment group. (F) Sections were stained with anti-CD31 antibodies to visualize tumor blood vessels (red), and counterstained with DAPI (blue). (G) Analysis of blood vessels in tumor tissues stained with anti-CD31 antibody (N=3).

$1 \mathrm{mg} / \mathrm{kg}$ every 3.5 days, for eight doses with a total cumulative dose of $8 \mathrm{mg} / \mathrm{kg}$. By day 28, administration of SP94-LD had significantly inhibited tumor growth by $74.6 \%(\mathrm{P}<0.01)$, whereas treatment with LD and FD inhibited tumor growth by $56.1(\mathrm{P}<0.01)$ and 11.6\% $(\mathrm{P}>0.05)$, respectively (Fig. 5A and $\mathrm{B})$, as compared to untreated controls. SP94-LD-mediated inhibition of growth was more significant than that mediated by LD ( $\mathrm{P}=0.013)$. The SP94-LD and LD groups did not exhibit significant changes in body weight (Fig. 5C) or complete blood counts (Table I) during the treatment period.
Histological analysis of the SK-HEP-1 tumors revealed that FD, LD, and SP94-LD induced apoptosis (Fig. 5D and E), with SP94-LD treatment resulting in the greatest proportion of apoptotic cells (Fig. 5E). The CD31 (angiogenesis) index (Fig. 5F and G) of SP94-LD treated tumors was lower compared to that of tumors treated with FD, LD, or saline.

We have previously observed that SP94-peptide-targeted liposomal doxorubicin (SP94-LD) inhibits the proliferation of small tumors. Nevertheless, in many cancer cases in humans, the tumors are detected when they are large. Thus, 
A
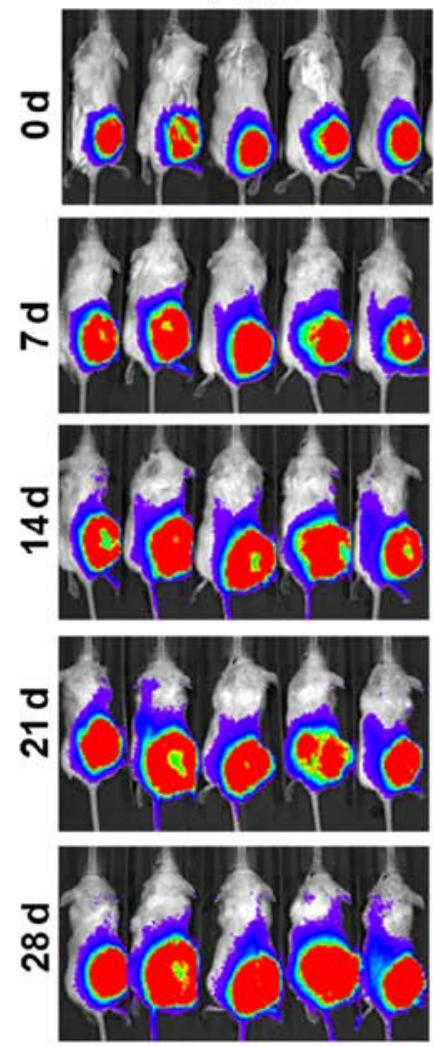

FD
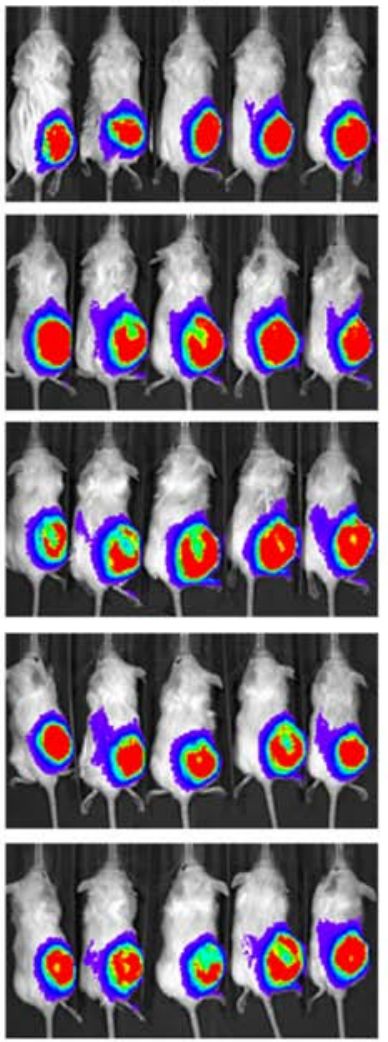

LD
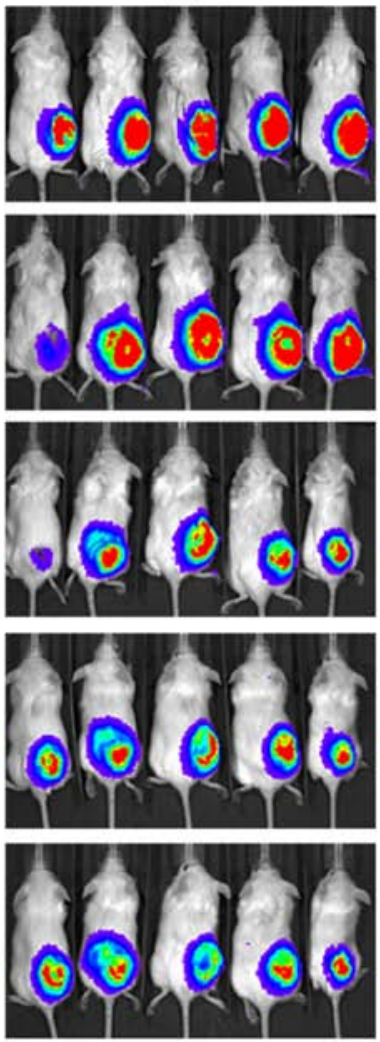

SP94-LD
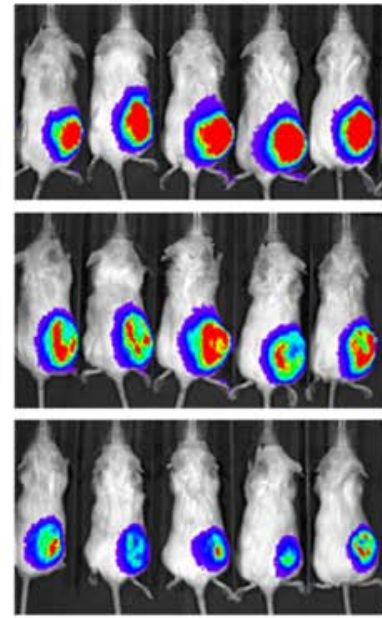

$-100$

$-80 \frac{x}{0}$
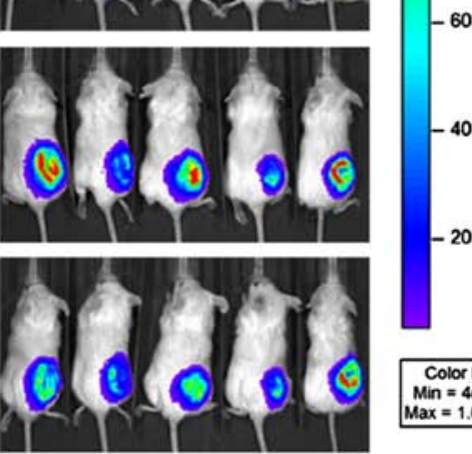

$-40$
B

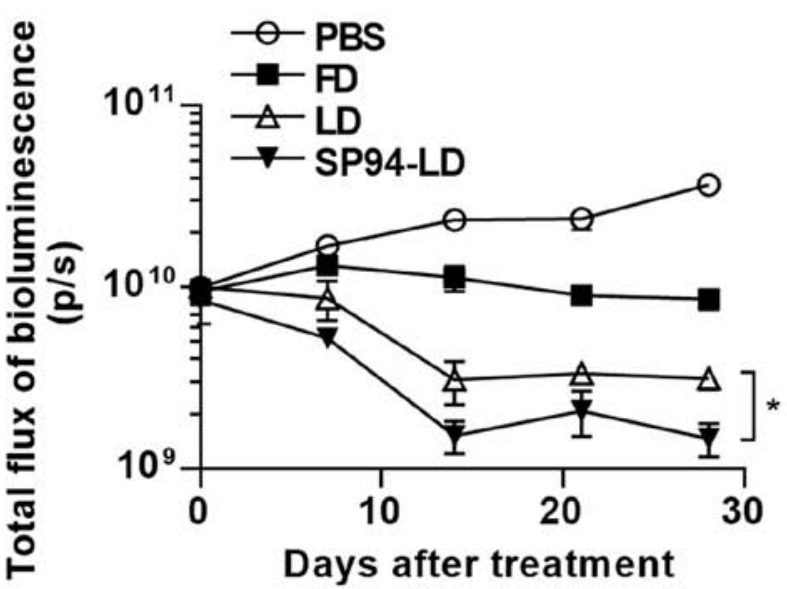

C

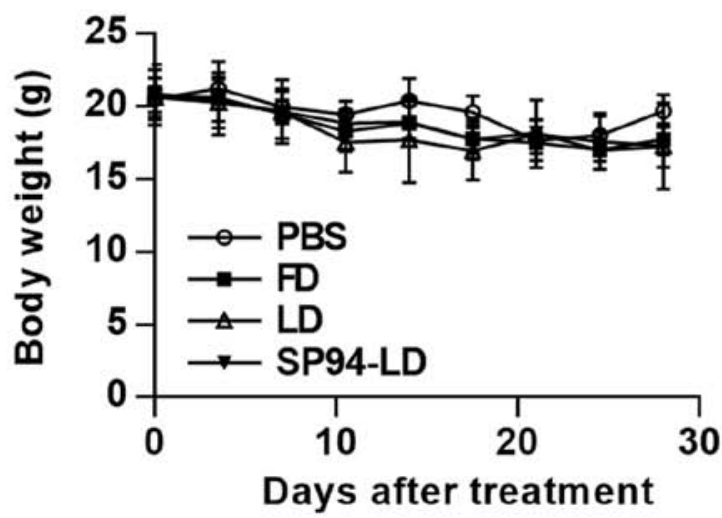

Figure 6. Antitumor capacity of liposomal doxorubicin conjugated to targeting peptide SP94 in animals with advanced hepatocellular carcinoma. (A) NOD. $\mathrm{CB} 17-P r k d c^{\mathrm{scid}} / \mathrm{J}$ mice were injected with $5 \times 10^{6} \mathrm{SK}-\mathrm{Hep}-1-$ Luc cells expressing luciferase. Tumor growth was examined by monitoring bioluminescence using the IVIS 200 Imaging system. All animals were kept under a constant supply of isoflurane using an automated anesthesia machine attached to an imaging device. After the total flux of bioluminescence reached $9.5 \times 10^{9} \mathrm{p} / \mathrm{s}$, mice were treated with $1 \mathrm{mg} / \mathrm{kg} \mathrm{SP94-LD,} 1 \mathrm{mg} / \mathrm{kg} \mathrm{LD}, 2 \mathrm{mg} / \mathrm{kg}$ FD, or PBS. All compounds were injected twice weekly via tail vein. (B) Vital tumor cells monitored by bioluminescence quantification. (C) Body weight of each group. P-values were calculated by t-test. ${ }^{*} \mathrm{P}<0.05$.

here we examined the activities of FD, LD, and SP94-LD against large tumors. Luciferase activity in live cells of mice bearing hepatocellular carcinoma xenografts was measured after i.v. injection with FD, LD, or SP94-LD (Fig. 6A). We observed a statistically significant reduction of tumor growth after i.v. treatment of hepatocellular carcinoma with SP94-LD, as compared with tumors in untreated and FD or LD-treated mice (Fig. 6A and B). SP94-LD significantly inhibited tumor growth as evidenced by a $91.47 \%$ reduction in luminescence $(\mathrm{P}<0.01)$, whereas treatment with $\mathrm{LD}$ reduced luminescence by
96.01\% ( $\mathrm{P}<0.01)$ compared with untreated controls. SP94-LD inhibition of growth was more significant than that by LD $(\mathrm{P}=0.011)$. Neither SP94-LD nor LD had a significant effect on body weight during the treatment period (Fig. 6C).

Effect of combination treatment with doxorubicin and vinorelbine in HCC cells. Previous study has shown that doxorubicin can inhibit HCC growth, but the clinical response was low. Combinations of anticancer agents with different mechanisms of action may be effective treatment regimens. 
Table I. Hematological parameters in NOS/SCID mice treated with different doxorubicin formulations.

\begin{tabular}{|c|c|c|c|c|}
\hline Parameter & SP94_LD & LD & FD & PBS \\
\hline WBC count & $3.10 \pm 0.43$ & $3.48 \pm 0.42$ & $7.16 \pm 0.75$ & $7.72 \pm 0.91$ \\
\hline NEU count & $2.36 \pm 0.41$ & $2.69 \pm 0.33$ & $5.95 \pm 0.63$ & $6.35 \pm 0.91$ \\
\hline LYM count & $0.17 \pm 0.01$ & $0.16 \pm 0.02$ & $0.54 \pm 0.05$ & $0.60 \pm 0.07$ \\
\hline RBC count & $6.29 \pm 0.06$ & $6.21 \pm 0.09$ & $7.72 \pm 0.22$ & $7.52 \pm 0.57$ \\
\hline HGB value & $10.55 \pm 0.22$ & $10.53 \pm 0.12$ & $13.20 \pm 0.43$ & $13.02 \pm 0.88$ \\
\hline HCT value & $31.32 \pm 1.08$ & $30.63 \pm 0.31$ & $38.82 \pm 1.29$ & $38.87 \pm 2.38$ \\
\hline RDW value & $19.40 \pm 0.43$ & $20.62 \pm 0.43$ & $23.05 \pm 0.51$ & $25.63 \pm 3.28$ \\
\hline PLT count & $1,172.83 \pm 85.13$ & $1,154.00 \pm 71.80$ & $940.17 \pm 57.09$ & $1,047.33 \pm 61.99$ \\
\hline MPV value & $7.89 \pm 0.12$ & $7.88 \pm 0.18$ & $6.79 \pm 0.11$ & $6.88 \pm 0.20$ \\
\hline PCT value & $0.92 \pm 0.06$ & $0.91 \pm 0.06$ & $0.64 \pm 0.04$ & $0.73 \pm 0.06$ \\
\hline NEU \% value & $74.63 \pm 2.82$ & $77.42 \pm 2.26$ & $83.13 \pm 1.78$ & $81.35 \pm 3.31$ \\
\hline LYM \% value & $5.22 \pm 0.78$ & $4.69 \pm 0.23$ & $7.86 \pm 1.06$ & $8.31 \pm 1.63$ \\
\hline MONO $\%$ value & $19.43 \pm 3.20$ & $16.97 \pm 1.68$ & $8.86 \pm 1.05$ & $10.06 \pm 2.09$ \\
\hline MONO count & $0.56 \pm 0.06$ & $0.60 \pm 0.11$ & $0.65 \pm 0.12$ & $0.75 \pm 0.15$ \\
\hline MCV value & $49.72 \pm 1.33$ & $49.33 \pm 0.51$ & $50.23 \pm 0.46$ & $52.22 \pm 2.16$ \\
\hline $\mathrm{MCH}$ value & $16.75 \pm 0.21$ & $16.93 \pm 0.16$ & $17.10 \pm 0.10$ & $17.43 \pm 0.47$ \\
\hline MCHC value & $33.80 \pm 0.64$ & $34.35 \pm 0.13$ & $34.07 \pm 0.20$ & $33.48 \pm 0.54$ \\
\hline PDW value & $18.12 \pm 0.19$ & $18.42 \pm 0.30$ & $18.90 \pm 0.57$ & $18.18 \pm 0.30$ \\
\hline
\end{tabular}
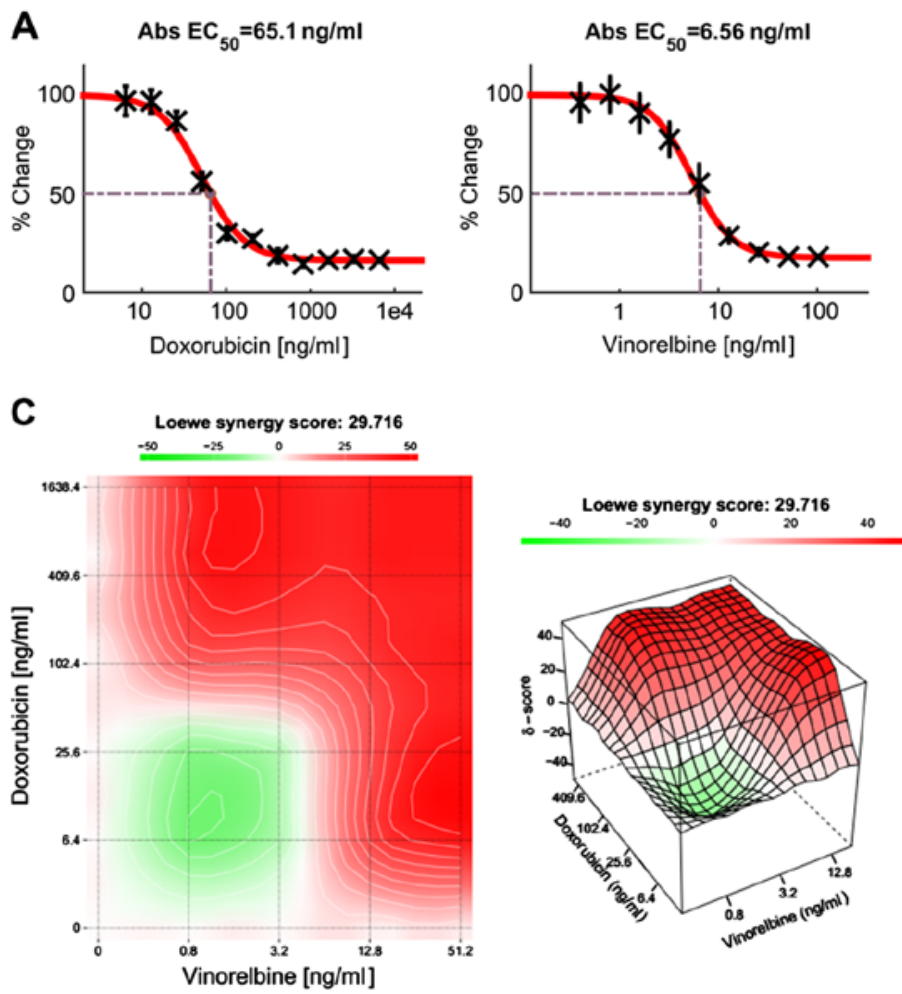
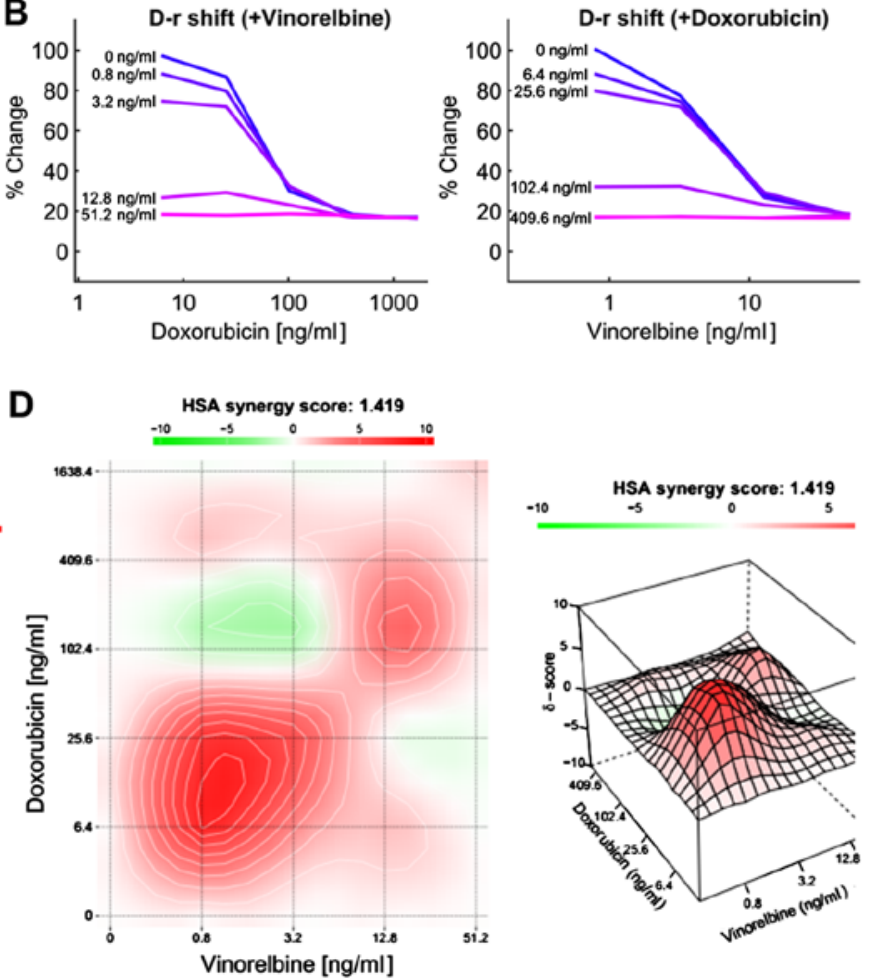

Figure 7. The combination of doxorubicin and vinorelbine in HCC cells. (A) Dose-response matrix for the effect of doxorubicin and vinorelbine in HCC cells. (B) Single-agent and combination responses determined by an MTT viability assay in Mahlavu cells. The landscapes of the combination responses for doxorubicin and vinorelbine based on (C) Loewe model and (D) HSA model.

For this reason, we attempted to develop an effective treatment for HCC patients by combining two or more anticancer drugs. In this study, HCC cells were treated with doxorubicin or vinorelbine alone, or in combination using a dose-matrix approach to evaluate the drug combination effects at various drug ratios. To determine the combination response (additivity, synergy, or antagonism), the Loewe Additivity score (27) and the Highest Single Agent (HAS) score (28) were calculated for the drug combination using the software Combenefit (24) and SynergyFinder (25). Fig. 7A presents a dose-response matrix 
between 6 concentrations of doxorubicin and 6 concentrations of vinorelbine in a 4-fold dilution scheme. The cytotoxicity of doxorubicin or/and vinorelbine over a wide range of molar ratios for HCC cells is shown in Fig. 7B. Vinorelbine significantly enhanced the effect of doxorubicin on the viability of HCC cells. Similarly, doxorubicin also enhanced the cytotoxicity of vinorelbine on HCC cells. The drug combination responses at various dose levels were quantified by computational methods. The dose-matrix combination showed the combination modeling between doxorubicin and vinorelbine by using the Loewe Additivity model (Fig. 7C) and HAS model (Fig. 7D). The synergy heatmaps showed that doxorubicin and vinorelbine have additive/synergistic effects (red areas in the model graph) on inhibiting cell proliferation at a wide range of drug combination ratio. These results suggest that the combined use of doxorubicin and vinorelbine may add an advantage to the current therapeutic regimens in liver cancer.

Therapeutic potential of combination therapy in an orthotopic hepatocellular carcinoma model. Models based on the subcutaneous injection of cancer cell lines may not accurately reproduce the biology of human HCC. To study the influence of the liver microenvironment upon response to therapy, we developed an orthotopic liver cancer model to recapitulate the tumor growth pattern seen in liver cancer patients. We investigated the antitumor potential of i.v. administration of both SP94-targeted liposomal doxorubicin and liposomal vinorelbine to SK-HEP-1-Luc tumors stably expressing firefly luciferase. Orthotopic tumor growth was non-invasively monitored by bioluminescence imaging. Prior to the first therapeutic injection (4 days after tumor cell implantation), growing orthotopic tumors were found to be localized mainly at the liver (Fig. 8A).

Mice were treated with either vehicle alone (PBS), FD $(1 \mathrm{mg} / \mathrm{kg})+$ free vinorelbine $11(\mathrm{FV})(2 \mathrm{mg} / \mathrm{kg})$, $\mathrm{LD}(1 \mathrm{mg} / \mathrm{kg})+$ stable liposomal vinorelbine $(\mathrm{sLV})(2 \mathrm{mg} / \mathrm{kg})$, SP94-LD $(1 \mathrm{mg} / \mathrm{kg})+$ SP94-sLV $(2 \mathrm{mg} / \mathrm{kg})$, or sorafenib alone (30 mg/kg) every other day for sixteen days. Bioluminescence was examined weekly to monitor tumor burden. Bioluminescence images revealed significant inhibition of tumor growth in the SP94-LD (1 mg/kg) + SP94-sLV (2 mg/kg)treated group compared with the vehicle control group, the FD $(1 \mathrm{mg} / \mathrm{kg})+\mathrm{FV}(2 \mathrm{mg} / \mathrm{kg})$ group, or the sorafenib $(30 \mathrm{mg} / \mathrm{kg})$ group (Fig. 8A and B). Body weight was not affected by any treatment regimen (Fig. 8C). Kaplan-Meier survival curves of all groups are shown in Fig. 8D. At the end of the study, the median survival times for the PBS, FD + FV, LD + sLV, SP94-LD + SP94-sLV, and sorafenib treatment groups were 39.5, 20, 69, 75 and 41.5 days, respectively (Fig. 8D and E). A survival analysis with a log-rank (Mantel-Cox) test revealed that SP94-LD + SP94-sLV treatment significantly extended animal survival as compared with PBS, FD + FV, LD + sLV, or sorafenib treatment (Fig. $8 \mathrm{~F}$ ).

\section{Discussion}

Lipid nanocarriers have been widely used to increase the efficacy of chemotherapeutics, largely through passive accumulation achieved by the enhanced permeability and retention effect (29). Additionally, their specificity and internalization by target tissues can be further enhanced by surface conjugation with targeting moieties (29-31). Nevertheless, insufficient differential affinity of targeting moieties between tumor and normal tissues remains a critical challenge for clinical application. This problem may arise because the molecules recognized by targeting moieties are not only expressed by tumor cells, but also by normal tissue. Therefore, it is of great importance to study the biodistribution and targeting potential of targeted nanocarriers.

Difficulties in developing specific imaging methods for HCC are caused by the lack of specific molecular targets, problems with drug delivery, and poor signal-to-noise ratios. It was previously reported that near-infrared, fluorochromelabeled phage probes allow for cancer targeting and imaging in vivo $(22,32)$. In this study, we used near-infrared fluorochrome-labeled PC94 phage and in vivo fluorescence imaging techniques, which enabled us to study in vivo tumor targeting and tissue distribution of SP94 peptide over time in the living animal. The HiLyte Fluor 750-labeled PC94 phage was able to bind to HCC and exhibited more rapid tumor localization than the control, as evidenced by fluorescence images of the tumor ex vivo and in living mice. Both HiLyte Fluor 750-labeled PC94 phage and control phage accumulated rapidly at high concentrations in liver, spleen, and bone marrow, indicating that the mononuclear phagocytes of the reticuloendothelial system (RES) are involved in the clearance of some of the circulating phages in mice. Although the tumor-to-background ratio was satisfactory in this study, the uptake of near-infrared fluorochrome-labeled phages by the RES is, nonetheless, quite obvious.

To investigate the potential of SP94 peptide as a target ligand for the delivery of therapeutics to tumors, we used this peptide to modify a clinically-used PEGylated liposomal drug through post-insertion technology, and examined the resulting pharmacokinetic profile and biodistribution in vivo. This post-insertion technique facilitates efficient insertion of ligand-PEG-DSPE conjugates into preformed liposomes under the right conditions (33). The plasma pharmacokinetics of SP94-LD was found to be indistinguishable from that of LD, indicating that the SP94 peptide conjugation did not compromise circulation time or stability. Comparison of the biodistribution of SP94-LD versus LD at $24 \mathrm{~h}$ post-treatment showed that doxorubicin accumulation was greater in the tumor, and that accumulation was 1.6-fold greater for SP94-LDtreated as compared with LD-treated mice. The bioavailable drug concentrations of SP94-LD reached maximum levels by $24 \mathrm{~h}$ after injection, and SP94 targeting increased drug delivery to intracellular tumors by 1.8 -fold. While SP94-LD and LD have similar plasma circulation profiles, the presence of the SP94-targeting moiety enhances drug delivery to tumors and the bioavailability of drug in tumors. However, SP94 targeting did not affect doxorubicin accumulation in the brain, heart, lung, liver, or kidneys. These results indicate that SP94-LD may improve the therapeutic index by increasing drug accumulation in the tumor, but not in normal tissue.

The use of peptides as targeting ligands offers several advantages, including low immunogenicity, small size, ready diffusion, ease of manufacturing, and simple targeted formulation assembly, when compared to larger biomolecules, such as antibodies. Previous studies showed that 
A

PBS

$\mathrm{FD}+\mathrm{FV}$

LD+sLV

SP94LD+SP94-sLV

Sorafenib

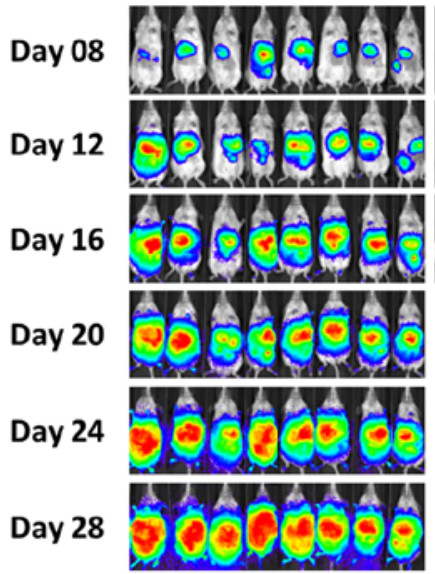

Day 32

Day 39

Day 59

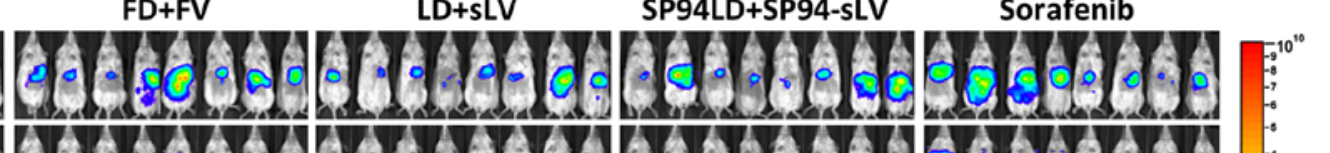

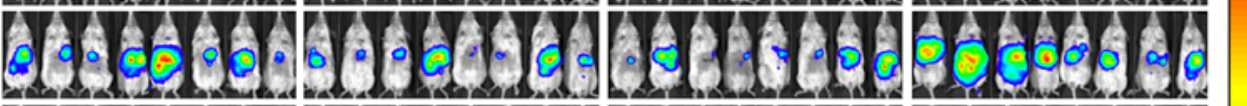
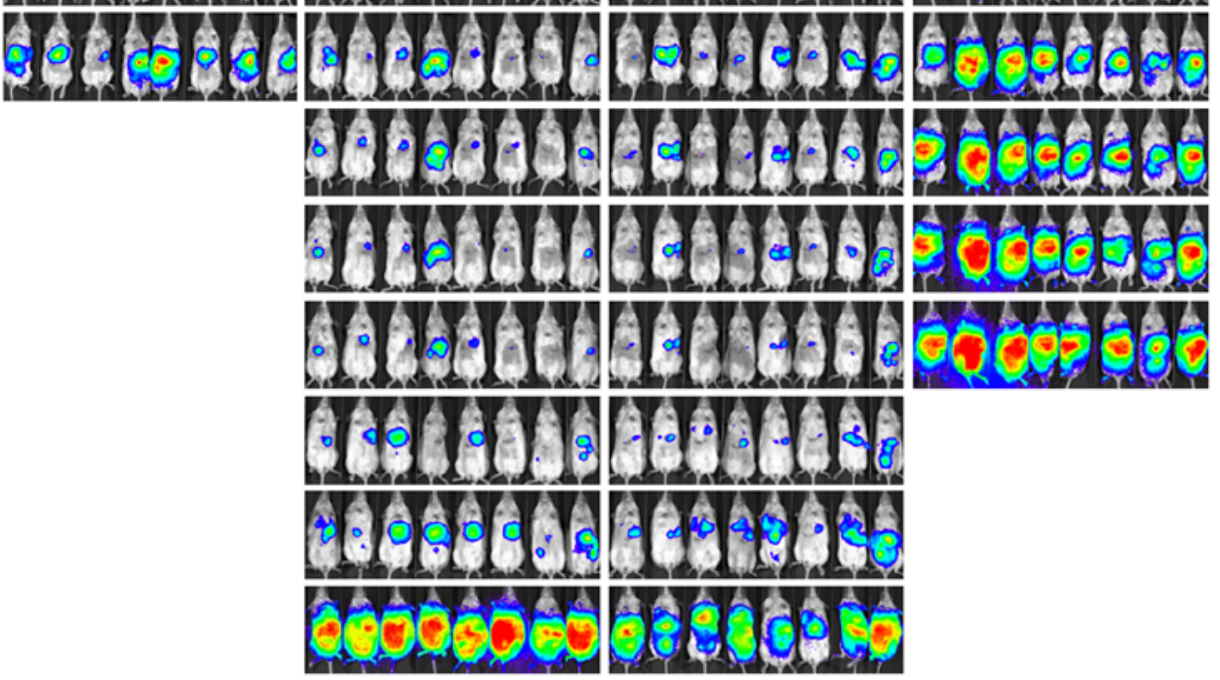

B

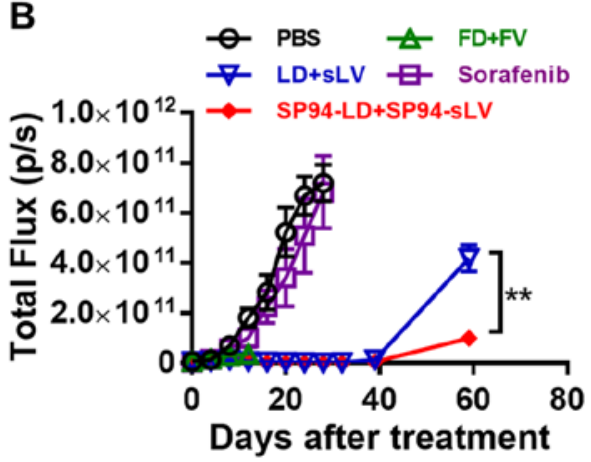

C

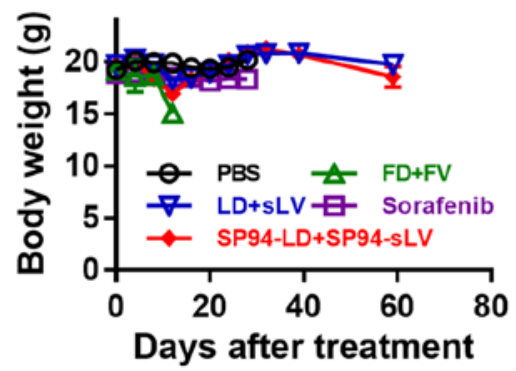

D

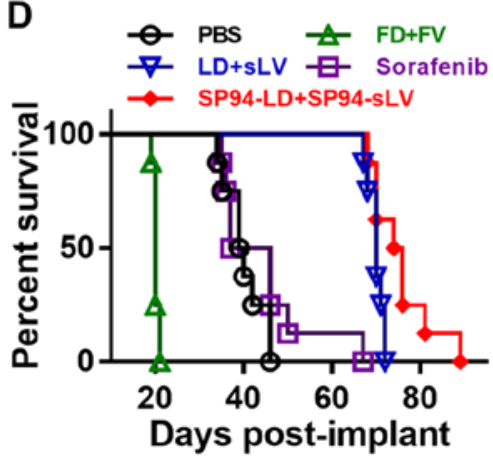

E

\begin{tabular}{cc} 
Group & $\begin{array}{c}\text { Median survival } \\
\text { (days) }\end{array}$ \\
\hline PBS & 39.5 \\
FD+FV & 20 \\
LD+sLV & 70 \\
SP94-LD+SP94-SLV & 75 \\
Sorafenib & 41.5
\end{tabular}

$\mathbf{F}$

Log-rank (Mantel-Cox) test

\begin{tabular}{|c|c|c|c|c|}
\hline & PBS & $F D+F V$ & $L D+s L V$ & Sorafenib \\
\hline SP94-LD+SP94-SLV & $<0.0001$ (****) $^{*}$ & $<0.0001$ (****) & $0.0227(*)$ & $<0.0001$ (****) \\
\hline Sorafenib & $0.3166(\mathrm{~ns})$ & $\left.<0.00011^{(\star \star \star \star}\right)$ & $0.003(* \pi)$ & \\
\hline LD+sLV & 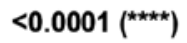 & $<0.0001$ (***) & & \\
\hline$F D+F V$ & $\left.<0.0001^{(\star \star \star \star}\right)$ & & & \\
\hline
\end{tabular}

Figure 8. Establishment of orthotopic models of hepatocellular carcinoma, and the therapeutic potential of combination therapy. NOD.CB17-Prkdc $c^{\text {sid } / J}$ mice were orthotopically implanted with SK-HEP-1-luc cells, and treated with vehicle (PBS), FD (1 mg/kg) $+\mathrm{FV}(2 \mathrm{mg} / \mathrm{kg}), \mathrm{LD}(1 \mathrm{mg} / \mathrm{kg})+\mathrm{sLV}(2 \mathrm{mg} / \mathrm{kg})$, SP94-LD $(1 \mathrm{mg} / \mathrm{kg})+$ SP94-sLV $(2 \mathrm{mg} / \mathrm{kg})$, or sorafenib $(30 \mathrm{mg} / \mathrm{kg})$, at 4 days after tumor inoculation. All compounds were injected every other day via tail vein. (A) Tumor growth was monitored based on bioluminescence, using the IVIS 200 Imaging system. (B) Kaplan-Meier survival analysis showing the probability of survival for all subjects. (C) Body weight. (D) Kaplan-Meier survival plot. (E) Median survival of each treatment group. (F) Survival analysis by log-rank (Mantel-Cox) test showing the probability of survival for all subjects $(\mathrm{N}=8)$.

using larger biomolecules as targeting ligands may increase the clearance of antibody-modified nanoparticles from the blood (34-36). This may be due to non-specific binding and uptake of nanoparticles by the RES (35). Our results showed that the modification of liposomes with SP94 peptide does not enhance immunogenicity, and has identical plasma pharmacokinetics as the original formulation. In addi- tion, SP94-mediated targeting enhanced tumor delivery by increasing overall tumor accumulation and cellular uptake, without affecting delivery to noncancerous host tissues or enhancing host toxicity. These findings were confirmed by in vivo near-infrared fluorescence imaging and biodistribution analysis. Previous studies with other targeted delivery systems have also suggested that targeting moieties increase 
drug accumulation in tumor tissues (37,38). However, targeted nanoparticles have not always caused a significant increase in overall tumor accumulation as compared to the non-targeted drugs. Previous studies showed that the use of macromolecule targeting ligands, such as antibodies (39) or transferrin (40), have a negligible impact on tumor accumulation and biodistribution. The differential effects of nanoparticles modified with macromolecules and those modified with small molecules may be due to differences in molecular size, affinity, and penetrability of the targeting ligand (41). It is possible that targeting moieties with high affinity would be subject to greater internalization and degradation by perivascular tumor cells, thereby limiting their penetration of tumors and reducing their tumor retention $(42,43)$.

At the time of writing, only sorafenib has been shown to exert a survival benefit in patients with hepatocellular carcinoma $(19,20)$. In our orthotopic liver cancer model, we found that sorafenib had no effect on tumor growth. FD + FV showed antitumor growth effects compared with PBS control group at day 8, 12 and 16 (Fig. 8A). However, the combination drugs induced the toxicity by markedly decreasing their body weights, leading to the death of the mice (Fig. 8C). LD + LV showed significant antitumor growth effects and reduced drug toxicity. SP94-LD + SP94-LV exhibited even higher therapeutic efficacy than LD + LV in an orthotopic model of human HCC, by reducing tumor size and prolonging the overall survival rate of the mice (Fig. 8). SP94-LD + SP94-sLV combination therapy shows strong clinical potential for treatment of HCC.

In conclusion, SP94-modification of liposomal doxorubicin significantly improves therapeutic efficacy in human

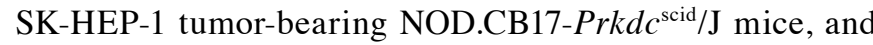
significantly inhibits tumor cell viability, resulting in reduced tumor volumes and final average tumor weights compared with control nonspecific treatments. The rapid, massive, and specific accumulation of SP94-LD in tumor cells results in prominent tumor growth regression. These therapeutic outcomes confirm the key role of the tumor-specific binding and internalization of SP94-LD in achieving elevated local concentrations of chemotherapeutic agents inside tumors. These findings suggest a potential clinical benefit from SP94-targeted liposomal doxorubicin and liposomal vinorelbine combination therapy.

In conclusion, we have evaluated whether it is worth considering SP94-LD for use in clinical trials against liver cancer. SP94-LD caused significantly greater inhibition of human hepatocellular carcinoma xenograft tumor growth when compared with LD, without increasing toxicity. SP94-LD has similar plasma pharmacokinetics as LD. Moreover, SP94-mediated targeting enhances therapeutic efficacy in the hepatocellular carcinoma xenograft mouse model by improving tumor pharmacokinetics and tissue distribution, allowing large amounts of antitumor drug to accumulate in tumors. These results indicate that SP94-mediated targeting for cancer therapy has distinct advantages over standard LD. Furthermore, it provides a promising opportunity to further improve the therapeutic index of the original formulation, which can be rapidly translated into the clinic given the availability of LD. Our results should also encourage further research to expand the application of this targeting ligand to various other drug-delivery nanoparticles.

\section{Acknowledgements}

The authors thank the Core Facility of the Institute of Cellular and Organismic Biology for their technical assistance. This study was supported by grants from Academia Sinica and Ministry of Science and Technology (MOST 104-0210-01-0902, MOST 105-0210-01-13-01, MOST 106-2623-E-001-001 and MOST 106-0210-01-15-02), Taiwan (to H.-C. Wu).

\section{References}

1. Souto E (ed): Lipid Nanocarriers in Cancer Diagnosis and Therapy. iSmithers Rapra Publishing, Shrewsbury, 2011.

2. Maeda H, Wu J, Sawa T, Matsumura Y and Hori K: Tumor vascular permeability and the EPR effect in macromolecular therapeutics: A review. J Controlled Release 65: 271-284, 2000.

3. Wu CH, Liu IJ, Lu RM and Wu HC: Advancement and applications of peptide phage display technology in biomedical science. J Biomed Sci 23: 8, 2016.

4. Lopes de Menezes DE, Pilarski LM and Allen TM: In vitro and in vivo targeting of immunoliposomal doxorubicin to human B-cell lymphoma. Cancer Res 58: 3320-3330, 1998.

5. Lu RM, Chang YL, Chen MS and Wu HC: Single chain anti-c-Met antibody conjugated nanoparticles for in vivo tumor-targeted imaging and drug delivery. Biomaterials 32: 3265-3274, 2011

6. Farokhzad OC, Jon S, Khademhosseini A, Tran TN, Lavan DA and Langer R: Nanoparticle-aptamer bioconjugates: A new approach for targeting prostate cancer cells. Cancer Res 64: 7668-7672, 2004.

7. Gabizon A, Shmeeda H, Horowitz AT and Zalipsky S: Tumor cell targeting of liposome-entrapped drugs with phospholipidanchored folic acid-PEG conjugates. Adv Drug Deliv Rev 56: 1177-1192, 2004.

8. Lee RJ and Low PS: Folate-mediated tumor cell targeting of liposome-entrapped doxorubicin in vitro. Biochim Biophys Acta 1233: 134-144, 1995.

9. Lee TY, Wu HC, Tseng YL and Lin CT: A novel peptide specifically binding to nasopharyngeal carcinoma for targeted drug delivery. Cancer Res 64: 8002-8008, 2004.

10. Lo A, Lin CT and Wu HC: Hepatocellular carcinoma cellspecific peptide ligand for targeted drug delivery. Mol Cancer Ther 7: 579-589, 2008.

11. Wu $\mathrm{CH}$, Kuo YH, Hong RL and Wu HC: $\alpha$-Enolase-binding peptide enhances drug delivery efficiency and therapeutic efficacy against colorectal cancer. Sci Transl Med 7: 290ra91, 2015.

12. Yeh CY, Hsiao JK, Wang YP, Lan CH and Wu HC: Peptideconjugated nanoparticles for targeted imaging and therapy of prostate cancer. Biomaterials 99: 1-15, 2016.

13. Chen WC, Completo GC, Sigal DS, Crocker PR, Saven A and Paulson JC: In vivo targeting of B-cell lymphoma with glycan ligands of CD22. Blood 115: 4778-4786, 2010.

14. Ashley CE, Carnes EC, Phillips GK, Padilla D, Durfee PN, Brown PA, Hanna TN, Liu J, Phillips B, Carter MB, et al: The targeted delivery of multicomponent cargos to cancer cells by nanoporous particle-supported lipid bilayers. Nat Mater 10: 389-397, 2011.

15. Ashley CE, Carnes EC, Epler KE, Padilla DP, Phillips GK, Castillo RE, Wilkinson DC, Wilkinson BS, Burgard CA, Kalinich RM, et al: Delivery of small interfering RNA by peptide-targeted mesoporous silica nanoparticle-supported lipid bilayers. ACS Nano 6: 2174-2188, 2012.

16. Ashley CE, Carnes EC, Phillips GK, Durfee PN, Buley MD, Lino CA, Padilla DP, Phillips B, Carter MB, Willman CL, et al: Cell-specific delivery of diverse cargos by bacteriophage MS2 virus-like particles. ACS Nano 5: 5729-5745, 2011.

17. Li Y, Hu Y, Xiao J, Liu G, Li X, Zhao Y, Tan H, Shi H and Cheng D: Investigation of SP94 peptide as a specific probe for hepatocellular carcinoma imaging and therapy. Sci Rep 6: 33511, 2016.

18. Zhang X, Ng HLH, Lu A, Lin C, Zhou L, Lin G, Zhang Y, Yang $Z$ and Zhang H: Drug delivery system targeting advanced hepatocellular carcinoma: Current and future. Nanomedicine (Lond) 12: 853-869, 2016.

19. Villanueva A, Hernandez-Gea V and Llovet JM: Medical therapies for hepatocellular carcinoma: A critical view of the evidence. Nat Rev Gastroenterol Hepatol 10: 34-42, 2013. 
20. Bruix J, Gores GJ and Mazzaferro V: Hepatocellular carcinoma: Clinical frontiers and perspectives. Gut 63: 844-855, 2014.

21. Jaye DL, Geigerman CM, Fuller RE, Akyildiz A and Parkos CA: Direct fluorochrome labeling of phage display library clones for studying binding specificities: Applications in flow cytometry and fluorescence microscopy. J Immunol Methods 295: 119-127, 2004.

22. Kelly KA, Waterman P and Weissleder R: In vivo imaging of molecularly targeted phage. Neoplasia 8: 1011-1018, 2006.

23. Laginha KM, Verwoert S, Charrois GJ and Allen TM: Determination of doxorubicin levels in whole tumor and tumor nuclei in murine breast cancer tumors. Clin Cancer Res 11: 6944-6949, 2005.

24. Di Veroli GY, Fornari C, Wang D, Mollard S, Bramhall JL, Richards FM and Jodrell DI: Combenefit: An interactive platform for the analysis and visualization of drug combinations. Bioinformatics 32: 2866-2868, 2016.

25. Ianevski A, He L, Aittokallio T and Tang J: SynergyFinder: A web application for analyzing drug combination dose-response matrix data. Bioinformatics 33: 2413-2415, 2017

26. Woodcock J and Woosley R: The FDA critical path initiative and its influence on new drug development. Annu Rev Med 59: 1-12, 2008.

27. Loewe $S$ : The problem of synergism and antagonism of combined drugs. Arzneimittelforschung 3: 285-290, 1953.

28. Berenbaum MC: What is synergy? Pharmacol Rev 41: 93-141, 1989.

29. Torchilin VP: Passive and active drug targeting: Drug delivery to tumors as an example. Handb Exp Pharmacol 197: 3-53, 2010.

30. Mohanty C, Das M, Kanwar JR and Sahoo SK: Receptor mediated tumor targeting: An emerging approach for cancer therapy. Curr Drug Deliv 8: 45-58, 2011.

31. Brown KC: Peptidic tumor targeting agents: The road from phage display peptide selections to clinical applications. Curr Pharm Des 16: 1040-1054, 2010

32. Hilderbrand SA, Kelly KA, Niedre M and Weissleder R: Near infrared fluorescence-based bacteriophage particles for ratiometric pH imaging. Bioconjug Chem 19: 1635-1639, 2008.

33. Moreira JN, Ishida T, Gaspar R and Allen TM: Use of the postinsertion technique to insert peptide ligands into pre-formed stealth liposomes with retention of binding activity and cytotoxicity. Pharm Res 19: 265-269, 2002.

34. Harding JA, Engbers CM, Newman MS, Goldstein NI and Zalipsky S: Immunogenicity and pharmacokinetic attributes of poly(ethylene glycol)-grafted immunoliposomes. Biochim Biophys Acta 1327: 181-192, 1997.
35. Koning GA, Kamps JA and Scherphof GL: Interference of macrophages with immunotargeting of liposomes. J Liposome Res 12: 107-119, 2002.

36. ElBayoumi TA and Torchilin VP: Tumor-targeted nanomedicines: enhanced antitumor efficacy in vivo of doxorubicin-loaded, longcirculating liposomes modified with cancer-specific monoclonal antibody. Clin Cancer Res 15: 1973-1980, 2009.

37. Liu Z, Cai W, He L, Nakayama N, Chen K, Sun X, Chen X and Dai $\mathrm{H}$ : In vivo biodistribution and highly efficient tumour targeting of carbon nanotubes in mice. Nat Nanotechnol 2: 47-52, 2007.

38. Qian X, Peng XH, Ansari DO, Yin-Goen Q, Chen GZ, Shin DM, Yang L, Young AN, Wang MD and Nie S: In vivo tumor targeting and spectroscopic detection with surface-enhanced Raman nanoparticle tags. Nat Biotechnol 26: 83-90, 2008.

39. Kirpotin DB, Drummond DC, Shao Y, Shalaby MR, Hong K, Nielsen UB, Marks JD, Benz CC and Park JW: Antibody targeting of long-circulating lipidic nanoparticles does not increase tumor localization but does increase internalization in animal models. Cancer Res 66: 6732-6740, 2006.

40. Bartlett DW, Su H, Hildebrandt IJ, Weber WA and Davis ME: Impact of tumor-specific targeting on the biodistribution and efficacy of siRNA nanoparticles measured by multimodality in vivo imaging. Proc Natl Acad Sci USA 104: 15549-15554, 2007.

41. Moghimi SM, Hunter AC and Andresen TL: Factors controlling nanoparticle pharmacokinetics: An integrated analysis and perspective. Annu Rev Pharmacol Toxicol 52: 481-503, 2012.

42. Adams GP, Schier R, McCall AM, Simmons HH, Horak EM, Alpaugh RK, Marks JD and Weiner LM: High affinity restricts the localization and tumor penetration of single-chain fv antibody molecules. Cancer Res 61: 4750-4755, 2001.

43. Rudnick SI, Lou J, Shaller CC, Tang Y, Klein-Szanto AJ, Weiner LM, Marks JD and Adams GP: Influence of affinity and antigen internalization on the uptake and penetration of Anti-HER 2 antibodies in solid tumors. Cancer Res 71: 2250-2259, 2011

(i) $\Theta$ This work is licensed under a Creative Commons Attribution-NonCommercial-NoDerivatives 4.0 International (CC BY-NC-ND 4.0) License. 